\title{
LA SELVA BAJA CADUCIFOLIA EN UNA CORRIENTE DE LAVA VOLCÁNICA EN EL CENTRO DE VERACRUZ: LISTA FLORÍSTICA DE LA FLORA VASCULAR
}

\author{
Gonzalo Castillo-Campos ${ }^{1,4}$, Patricia Dávila-Aranda ${ }^{2}$ y José Alejandro Zavala-Hurtado ${ }^{3}$
}

\author{
'Departamento de Biodiversidad y Sistemática, Instituto de Ecología, A.C., \\ km 2.5 Carretera Antigua a Coatepec No. 351, Congregación El Haya, Xalapa 91070, Veracruz, México. \\ ${ }^{2}$ Facultad de Estudios Superiores Iztacala, Universidad Nacional Autónoma de México, \\ Av. de Los Barrios No. 1, Los Reyes Iztacala, Tlalnepantla 54090, Estado de México, México. \\ ${ }^{3}$ Departamento de Biología, Universidad Autónoma Metropolitana-Iztapalapa, \\ Apdo. Postal 55-535, México 09340, D.F., México. \\ ${ }^{4}$ Autor para la correspondencia; correo-e: gonzalo.castillo@inecol.edu.mx; Tel (228) 842-1825, Fax (228) 818-7809.
}

\begin{abstract}
Resumen: El objetivo del presente trabajo fue elaborar un inventario florístico de la vegetación original y secundaria de la selva baja caducifolia (SBC) sobre sustratos rocosos o corrientes de lava volcánica que han sido escasamente estudiados y se encuentran en buen estado de conservación, en el centro del estado de Veracruz. A través de transectos en un gradiente altitudinal de 400 a $900 \mathrm{~m}$, se muestrearon áreas de $1,000 \mathrm{~m}^{2}$ para la vegetación primaria y secundaria a cada $100 \mathrm{~m}$ de altitud, donde se recolectaron las especies presentes. Se encontraron 104 familias con 394 géneros y 666 especies y subespecies de plantas vasculares. Las especies y subespecies presentaron los siguientes grupos de forma de crecimiento: 88 árboles, 126 arbustos, 342 hierbas, 80 bejucos y 30 epífitas. Del total de las especies registradas, entre las familias más ricas en especies destacan Fabaceae, Asteraceae, Poaceae y Euphorbiaceae. Fue notable la presencia de un componente suculento en la flora de la SBC, conformada por 204 especies y subespecies, los cuales correspondieron a 30.4\% del total para estos taxa vasculares registrados en la zona de estudio. La mayor riqueza de especies suculentas se presentó en las familias más diversas, tales como Euphorbiaceae, Orchidaceae, Cactaceae y Bromeliaceae. En conclusión, se puede decir que la SBC del sustrato rocoso es muy rica en especies de la flora vascular, destacando el estrato herbáceo como el más diverso en ambas comunidades vegetales.
\end{abstract}

Palabras clave: centro de Veracruz, conservación, flora suculenta, flora vascular, México, riqueza.

\begin{abstract}
Currently, the tropical dry forest found on rock formations originating from volcanic activity are the best preserved, but also the least studied in the State of Veracruz, Mexico. We recorded 104 families comprising 394 genera and 666 species and subspecies of vascular plants. These latter taxa were classified in the following growth form categories: 88 trees, 126 shrubs, 342 herbs, 80 vines and 30 epiphytes. The richest families, considering the recorded species, were Fabaceae, Poaceae, Asteraceae and Euphorbiaceae. The presence of a rich succulent component in the tropical dry forest flora was noticeable. It is conformed by 204 species and subspecies, corresponding to $30.4 \%$ of the total for such vascular taxa recorded in the study area. The highest richness of succulent species was found in the most diverse families, such as Euphorbiaceae, Orchidaceae, Cactaceae, and Bromeliaceae.
\end{abstract}

Key words: central Veracruz, floristics, Mexico, tropical dry forest, volcanic lava field.

$\mathbf{V}$ eracruz destaca por ser uno de los estados más diversos de México, después de Chiapas y Oaxaca (Rzedowski, 1991). Éste es también uno de los estados mejor conocidos florísticamente. Sin embargo, las áreas de sustratos rocosos y los acantilados del estado son los hábitats menos conocidos en términos de su flora vascular.
También, es en este tipo de hábitats del centro de Veracruz donde la selva baja caducifolia (SBC) se encuentra en mejor estado de conservación y presenta una alta riqueza y diversidad florística (Cházaro, 1992).

La SBC está amplia e ininterrumpidamente distribuida en la vertiente pacífica de México, incluyendo la cuenca 
del río Balsas, donde se extiende desde el sur de Sonora y el suroeste de Chihuahua hasta Chiapas, continuándose hacia Centroamérica (Rzedowski, 1978). En la vertiente atlántica existen tres manchones aislados: el primero al sur de Tamaulipas, sureste de San Luis Potosí y extremo norte de Veracruz; el segundo en el centro de Veracruz; y el tercero en la parte norte de la Península de Yucatán, ocupando casi todo el estado de Yucatán y una fracción del de Campeche (Rzedowski, 1978; Sarukhán, 1998). La SBC en México es un tipo de vegetación que posee una gran riqueza de especies, inclusive en comparación con otras selvas secas neotropicales que se desarrollan en sitios con mejores condiciones de humedad (Toledo, 1982; Lott et al., 1987; Sarukhán, 1998; Pérez-García y Meave, 2004; Durán et al., 2006). Sin embargo, también se ha reportado que en condiciones ambientales extremas de temperatura y humedad baja, la riqueza y la diversidad de esta comunidad vegetal tienden a disminuir (Rzedowski y Calderón, 1987; Porembski et al., 1994; Silva et al., 1996).

En Veracruz, como en la mayoría de los estados del país, el grado de transformación de la SBC, producto de las actividades del hombre, varía de manera notable de una región a otra. El avance de este proceso está en función de varios factores, entre los cuales se encuentran los siguientes: (1) la capacidad del terreno para ser convertido en parcela agrícola o en agostadero, (2) el grado de utilidad de las especies de la vegetación natural, (3) el tipo, la intensidad y la duración de la acción humana ejercida, y (4) la resistencia intrínseca que ofrece una determinada comunidad vegetal a sufrir cambios profundos y su capacidad de regeneración (Rzedowski y Calderón, 1987).

La selva baja caducifolia que se desarrolla en hábitats rocosos presenta las mejores perspectivas de conservación, debido a que dichas áreas han sido las menos modificadas por las actividades humanas. Los sitios rocosos actualmente sirven de refugio para muchas especies de la biota original que han sido desplazadas de los ambientes aptos para el desarrollo de actividades agropecuarias. Entre la diversidad de especies originales de estos hábitats se encuentran las de distribución restringida o endémicas de Veracruz (CastilloCampos et al., 2005) y las que están en peligro de extinción, incluyendo algunas paleoendémicas, como las cícadas (Gómez-Pompa et al., 1994).

La SBC sensu Miranda y Hernández-X. (1963), localizada en la cuenca media-alta del río Actopan, en el centro del estado de Veracruz (INEGI, 1987a), ha sido poco estudiada desde el punto de vista florístico. Sin embargo, existen varios trabajos de este tipo que se han realizado para esta comunidad vegetal en otras áreas del centro del estado de Veracruz. Entre ellos podemos mencionar los siguientes: los de Ortega (1981) y Narave (1985), elaborados en la corriente de lava volcánica localizada al NE del Cofre de Perote; el de Acosta (1986), quien describe la estructura de la selva baja caducifolia desarrollada sobre el sustrato roco- so de la sierra de Manuel Díaz; el de Medina-Abreo y Castillo-Campos (1993), quienes listan las especies de la selva baja caducifolia en la barranca de Acazónica; y los de Castillo-Campos (1985, 1995), donde se describe la vegetación de los acantilados y sustratos calizos en el municipio de Jalcomulco. Además, es notable que la selva baja caducifolia sea una de las comunidades vegetales mejor representadas en los sustratos rocosos del centro de Veracruz (Ortega, 1981; Castillo-Campos, 1985; Acosta, 1986; Medina-Abreo y Castillo-Campos, 1993, 1996; CastilloCampos, 1995).

Los sustratos rocosos de origen volcánico son muy diversos, ya que incluyen tanto derrames de roca volcánica o malpaís, como sitios escarpados o acantilados de brechas volcánicas que se localizan desde la cima del Cofre de Perote hasta la línea costera del Golfo de México. Aunado a esto, las distintas condiciones climáticas han contribuido al desarrollo de diversas comunidades vegetales como la selva baja caducifolia, las selvas mediana subcaducifolia y subperennifolia, los encinares, el bosque mesófilo de montaña, los pinares, el matorral xerófilo, los pastizales y los páramos de altura (Gómez-Pompa, 1978).

Como se mencionó anteriormente, cabe acl a rar que en México el estudio de estos hábitats es escaso, por lo que es importante elaborar trabajos que contribuyan a su conocimiento, considerndo además que en la actualidad éstos son prácticamente los únicos sitios que no han sido drásticamente modificados por las actividades humanas (CastilloCampos, 1985, 1995; Acosta, 1986; Castillo-Campos, 2003). Algunos nuevos registros de especies de la flora vascular encontralos recientemente proceden de recolecciones realizadas en los sustratos rocosos del centro del estado de Veracruz (Castillo-Campos et al., 1998). Esto pone en evidencia el escaso conocimiento que se tiene de este tipo de hábitats. En las ex plo raciones más recientes de estos ambientes, se ha reportado que la selva baja caducifolia de los sustratos rocosos destaca por presentar el mayor porcentaje de especies endémicas, cuando menos para el centro del estado de Vera cruz (Castillo-Campos et al., 1999; Castillo-Campos, 2003; Castillo-Campos et al., 2005).

El objetivo de este trabajo fue preparar un inventario de la flora vascular, y describir y delimitar tipos de cobertura vegetal y de uso del suelo de una corriente de lava volcánica en el centro del estado de Veracruz.

\section{Materiales y método}

Área de estudio. El área de estudio está constituida por una corriente de lava volcánica o malpaís, que se encuentra en el fondo del valle plano de la cuenca media-alta del río Actopan, en el centro del estado de Ve ra c ruz. Se ubica entre las coordenadas $19^{\circ} 31^{\prime}$ y $19^{\circ} 37^{\prime}$ de latitud norte, y $96^{\circ} 41^{\prime}$ y $96^{\circ} 54^{\prime}$ de longitud oeste (figura 1). La zona tiene una forma alargada, con $17 \mathrm{~km}$ de longitud y una anch u ra varia- 


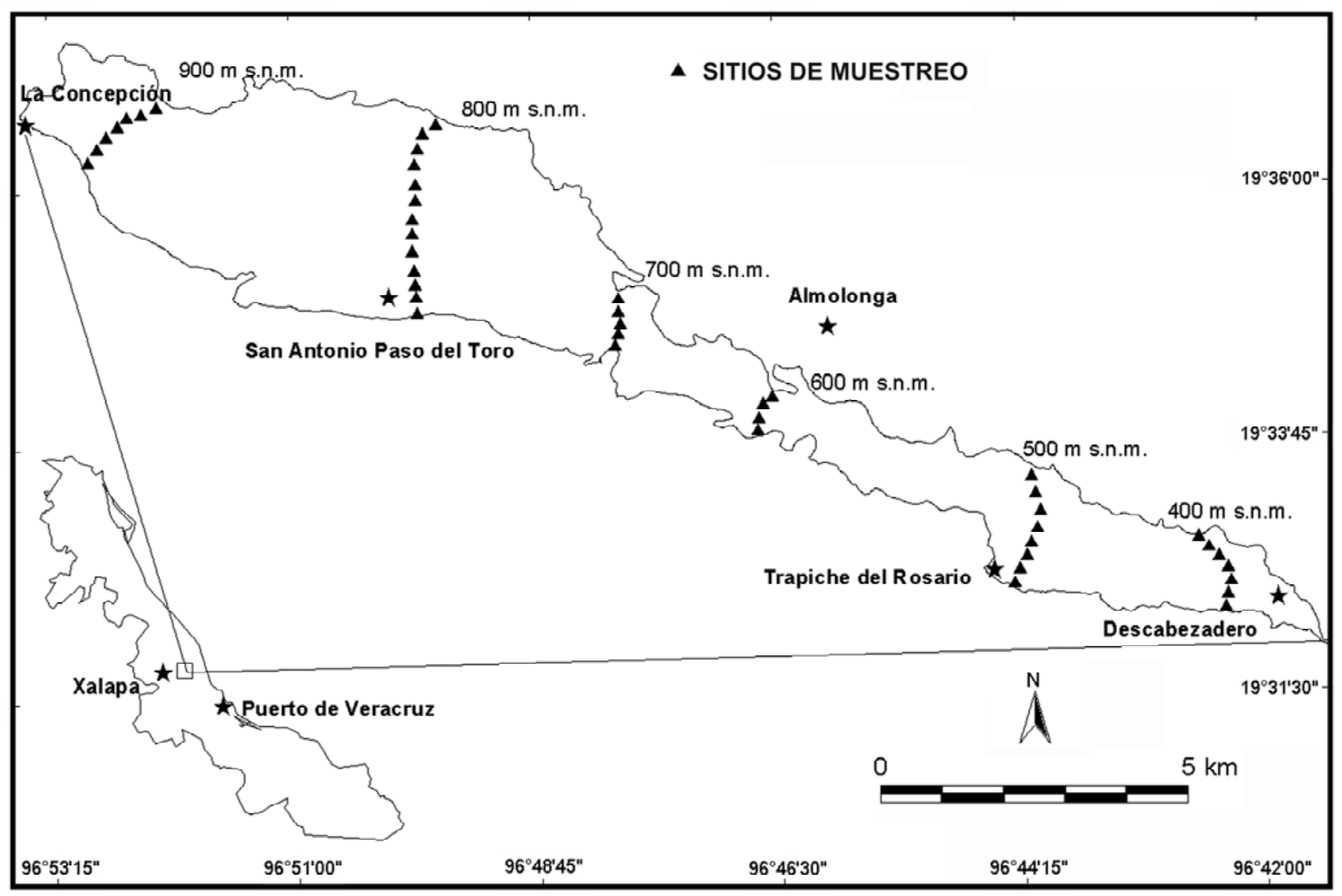

Figura 1. Localización de la zona de estudio y principales asentamientos humanos.

ble de entre 0.5 y $2.7 \mathrm{~km}$. Ocupa una superficie de 3,976 ha, la cual abarca parte de cuat romunicipios: Xalapa, Actopan, Emiliano Zap ata y Naolinco. Desde el punto de vista ge omorfológico, la zona pertenece a la Provincia Volcánica de las Faldas Bajas del Cofre de Pe rote y constituye un valle cubierto de basalto caótico y cenizas volcánicas con mat erial piro clástico poco consolidado (Rossignol, 1987), depositados en dife rentes períodos. Sin embargo, el derrame volcánico que ocupa la mayor extensión fue depositado en el Holoceno, hace ap roximadamente 10,000 años (Negendank et al., 1985). Los suelos son someros y están fo rmados básicamente por Litosoles, aunque los más profundos son B ru n i zems de maduración húmica, en proceso de planosolización y ve rtisolización (Rossignol y Geissert, 1987).

De acuerdo con los datos de la estación de Almolonga, localizada en la zona de estudio, el clima es del tipo Aw1(w), es decir, cálido subhúmedo con lluvias en verano, precipitación invernal menor de $5 \%$, con temperatura promedio de $22.3^{\circ} \mathrm{C}$, la mínima de $11^{\circ} \mathrm{C}$ y la máxima de $30^{\circ} \mathrm{C}$.

La precipitación total anual promedio es de 1,053.5 $\mathrm{mm}$ (figura 2) y presenta dos periodos bien marcados: el seco, de octubre a mayo, con una precipitación promedio mensual de $22 \mathrm{~mm}$ en marzo, y el lluvioso, de junio a sep-

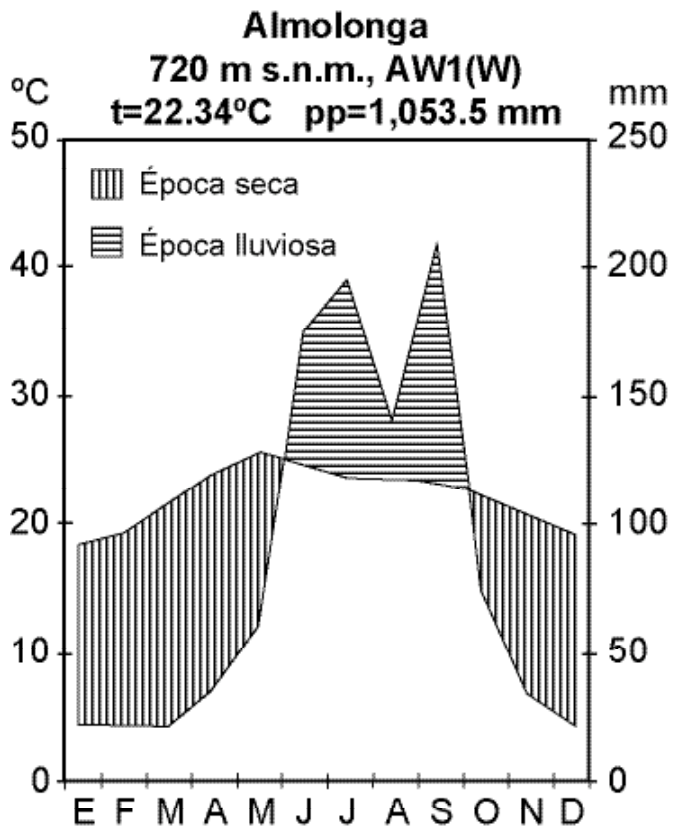

Figura 2. Diagrama ombrotérmico de Almolonga, Veracruz. 
tiembre, con un promedio mensual de $209 \mathrm{~mm}$ en septiembre (INEGI, 1987b; García, 1988).

Método. Con el empleo de mapas topográficos, fotografías aéreas y ortofotos escala 1:20,000, del año 1995, se delimitó el área de estudio y las unidades de vegetación y uso de suelo.

El inventario de las plantas vasculares se llevó a cabo en la vegetación establecida en el malpaís, en un gradiente altitudinal que va de 400 a $900 \mathrm{~m}$. Cada $100 \mathrm{~m}$ de altitud (figura 1) se trazaron cuadros de $100 \mathrm{~m}^{2}$ para el estrato arbóreo y arbustivo, y tres de $4 \mathrm{~m}^{2}$ al azar en el interior del cuadro más grande para el herbáceo, a intervalos ap roximados de $50 \mathrm{~m}$, siguiendo el nivel altitudinal. En promedio se muestrearon á reas de 2,000 $\mathrm{m}^{2}$ por transecto. El muestreo se realizó durante cuat ro meses consecutivos en dos años (agostonoviembre de 1999-2000), du rante la época lluviosa, que es cuando florecen y fructifican la mayoría de las especies.

En cada cuadro se recolectaron especímenes de las diferentes especies registradas. Se muestrearon como mínimo áreas de $1,000 \mathrm{~m}^{2}$ por transecto para cada comunidad vegetal (primaria y secundaria). El material botánico fue identificado en los herbarios XAL y MEXU, utilizando claves dicotómicas publicadas en la Flora de Veracruz y otras floras afines, y por comparación con material herborizado determinado por especialistas de los distintos grupos de plantas. El material determinado fue depositado en los herbarios MEXU (Universidad Nacional Autónoma de México), ENCB (Instituto Politécnico Nacional) y XAL (Instituto de Ecología, A.C.).

La nomenclatura utilizada para clasificar la vegetación presente en el área de estudio es la de Miranda y Hernández-X. (1963). El arreglo de las familias se hizo de acuerdo con el sistema de clasificación de Conquist (1988). La nomenclatura de los géneros y las especies fue verificada en la base de datos VAST del Missouri Botanical Garden (W'3 TROPICOS, consultada durante 2004). Los autores de las especies se abreviaron de acuerdo con Brummitt y Powell (1992). Todas las especies y subespecies incluidas en la lista fueron recolectadas por el primer autor de este trabajo.

Para la elaboración del mapa de vegetación y uso del suelo, se utilizaron ortofotos digitales del año 1995, usando como criterio principal el tono y la textura para la delimitación de las unidades de vegetación, las cuales fueron analizadas con el Sistema de Info rmación Geográfica ArcView 3.2a para Windows. Las unidades delimitadas se verificaron en campo con los muestreos de los inventarios florísticos.

\section{Resultados}

En las dos comunidades vegetales se recolectaron 1,700 números de ejemplares de plantas vasculares. De éstos, se identificaron 1,666, es decir, 98\%, y sólo quedó sin identificar $2 \%$ del material por carecer de estructuras reproductivas. Se registraron 104 familias con 394 géneros y 666 especies y subespecies (cuadro 1; apéndice 1); de éstos, 321 corresponden a la vegetación original y 346 a la vegetación secundaria (apéndice 1). Entre las especies y subespecies se presentaron las siguientes formas de crecimiento: 88 árboles, 126 arbustos, 342 hierbas, 80 bejucos y 30 epífitas (figura 3). Las lianas fueron agrupadas junto con los bejucos.

La flora suculenta de la selva baja caducifolia estuvo conformada por 33 familias, 91 géneros y 204 especies y subespecies, cifras correspondientes a $31.7 \%, 23 \%$ y $30.4 \%$ de sus respectivos totales registrados en la zona de estudio (cuadro 1). La mayor riqueza de especies suculentas se concentró en las familias más diversas del sistema, como Euphorbiaceae, Orchidaceae, Cactaceae y Bromeliaceae.

Descripción de la vegetación y uso del suelo. Se reconocieron dos tipos de comunidades vegetales, la selva baja caducifolia (SBC) primaria y la vegetación secundaria o acahual. Las dos comunidades vegetales ocuparon en conjunto una superficie ap roximada de 1,823 ha, que correspondió a $45 \%$ del total del área de estudio. El 55\% de la superficie restante del área estuvo ocupada por cultivos y pastizales, entrelos cuales destacaron por la superficie que ocuparon los de caña de azúcar con 1,204.08 ha, los pastizales inducidos e introducidos con 730.94 ha, los cultivos de mango con 211.66 ha y, muy por debajo de éstos, los cultivos de ch ayote con 75 ha y los de plátano con 8.01 ha (figura 4).

Selva baja caducifolia. La selva baja caducifolia sensu Miranda y Hernández-X. (1963) se distribuye de forma fragmentada en la mayor parte de la franja del malpaís. La superficie de los fragmentos observados varió desde menos de 1 ha $\left(2,922 \mathrm{~m}^{2}\right)$ hasta 952.50 ha. En total, la SBC ocupó una superficie de 1,387.20 ha, cifra que corresponde aproximadamente a $34 \%$ del área de estudio (figura 4 ) y presentó una riqueza de 321 especies y subespecies (48\% del total registrado para la zona de estudio). En una buena proporción, la selva baja caducifolia se encontró en buen estado de conservación (Ortega, 1981), principalmente en los derrames de basalto, donde la rocosidad ha limitado el pastoreo y el desarrollo de otras actividades agropecuarias.

Las especies que caracterizaron al estrato arbóreo de la SBC midieron entre 3 y $8 \mathrm{~m}$ de altura (figura 5). Este estrato estuvo dominado principalmente por Bursera cinerea, Cephalocereus palmeri var. sartorianus, Lysiloma microphyllum, L. acapulcensis y Pseudobombax ellipticum var. tenuiflorum. El estrato arbustivo estuvo caracterizado por Bemardia interrupta, Casearia corymbosa, Comocladia engleriana, Cnidoscolus aconitifolius, Croton ciliato-glandulosus, Diospyros oaxacana y Fraxinus schie - 


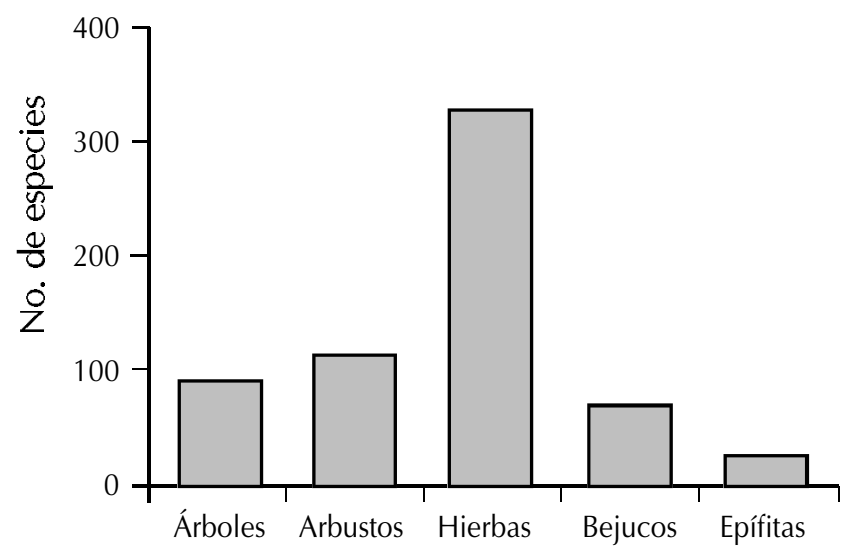

Figura 3. Riqueza de especies por formas de crecimiento registradas en la zona de estudio.

deana. Las especies características del estrato herbáceo fueron Anthurium schlechtendalii subsp. schlechtendalii, Bidens reptans, Callisia fragrans, $C$. repens, Mammillaria eriacantha, Microgramma nitida y Tillandsia fasciculata. En general, la SBC original se encontró bastante fragmentada y desplazada por pastizales y cultivos de caña, chayote, mango y café (figura 4).

En sentido estricto, la diversidad observada de especies epífitas en la SBC fue baja, y estuvo dominada y caracterizada principalmente por el género Tillandsia. Sin embargo, es importante señalar que en el mantillo orgánico acumulado entre las grietas del sustrato rocoso se han establecido en este hábitat una cierta diversidad de especies de orquídeas y bromelias, que en otras condiciones ambientales crecen como epífitas sobre especies arbóreas (figura 3; apéndice 1).

Vegetación secundaria. Aun con las limitantes propias del área de estudio, la selva baja caducifolia no ha escapado al impacto de las diversas actividades que realiza el hombre. Entre las actividades antrópicas que han modificado a la SBC de la zona se observaron los incendios provocados o escapados de las áreas de cultivo, el pastoreo de ganado caprino y vacuno, y la extracción de especies energéticas o con potencialidades de ornato como orquídeas, cactáceas, agaváceas, bromeliáceas y algunas fabáceas.

La vegetación secundaria, derivada de la antropización de la SBC, ocupó una superficie de 409.11 ha (figura 4) y presentó una riqueza de 346 especies y subespecies, correspondiente a $52 \%$ del total registrado. En la vegetación secundaria se reconocieron diferentes etapas de regeneración de acuerdo con la edad de abandono, desde las más

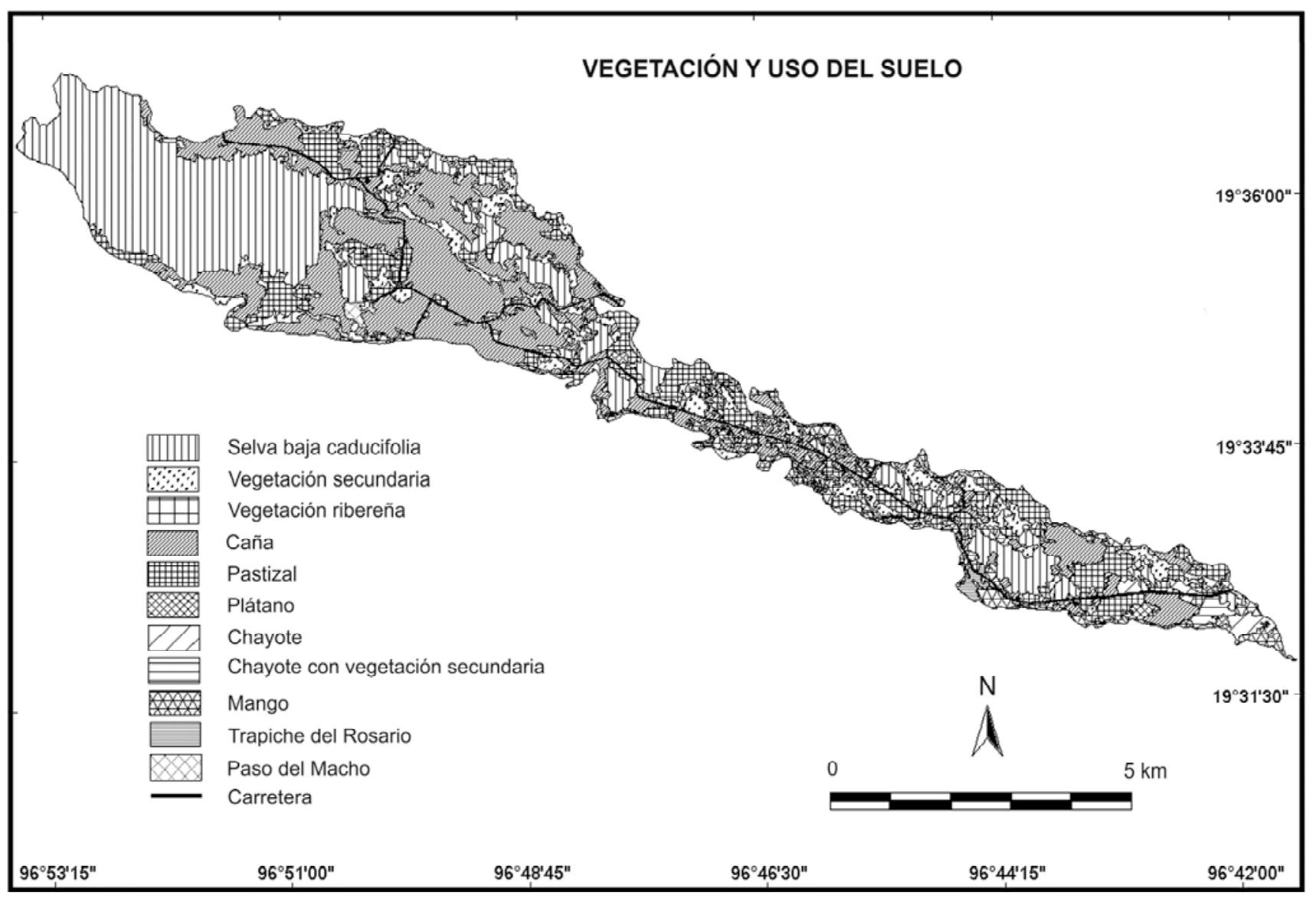

Figura 4. Vegetación y uso del suelo en la zona de estudio. 
Gonzalo Castillo-Campos, Patricia Dávila-Aranda y José Alejandro Zavala-Hurtado

Cuadro 1. Síntesis de la diversidad florística vascular de la zona de estudio, ordenada en forma decreciente por el número de especies de cada familia.

\begin{tabular}{|c|c|c|c|c|c|}
\hline \multirow[t]{2}{*}{ Familia } & \multicolumn{2}{|c|}{ Número de } & \multirow[t]{2}{*}{ Familia } & \multicolumn{2}{|c|}{ Número de } \\
\hline & Géneros & Especies & & Géneros & Especies \\
\hline Fabaceae & 35 & 76 & Capparidaceae & 1 & 2 \\
\hline Asteraceae & 45 & 65 & Dioscoreaceae & 1 & 2 \\
\hline Poaceae & 29 & 55 & Ebenaceae & 1 & 2 \\
\hline Euphorbiaceae & 13 & 45 & Loasaceae & 2 & 2 \\
\hline Malvaceae & 8 & 18 & Olacaceae & 2 & 2 \\
\hline Rubiaceae & 11 & 18 & Papaveraceae & 2 & 2 \\
\hline Bromeliaceae & 5 & 16 & Phytolaccaceae & 2 & 2 \\
\hline Cactaceae & 11 & 14 & Ranunculaceae & 1 & 2 \\
\hline Orchidaceae & 13 & 14 & Rutaceae & 2 & 2 \\
\hline Apocynaceae & 11 & 13 & Schizaeaceae & 1 & 2 \\
\hline Sapindaceae & 6 & 13 & Urticaceae & 2 & 2 \\
\hline Amaranthaceae & 6 & 12 & Vitaceae & 1 & 2 \\
\hline Boraginaceae & 4 & 12 & Annonaceae & 1 & 1 \\
\hline Solanaceae & 6 & 12 & Araliaceae & 1 & 1 \\
\hline Acanthaceae & 10 & 11 & Arecaceae & 1 & 1 \\
\hline Convolvulaceae & 4 & 11 & Aspleniaceae & 1 & 1 \\
\hline Labiatae & 4 & 11 & Betulaceae & 1 & 1 \\
\hline Verbenaceae & 8 & 11 & Brassicaceae & 1 & 1 \\
\hline Anacardiaceae & 7 & 9 & Campanulaceae & 1 & 1 \\
\hline Cyperaceae & 4 & 9 & Chenopodiaceae & 1 & 1 \\
\hline Tiliaceae & 4 & 9 & Cochlospermaceae & 1 & 1 \\
\hline Asclepiadaceae & 6 & 8 & Combretaceae & 1 & 1 \\
\hline Moraceae & 2 & 8 & Cunoniaceae & 1 & 1 \\
\hline Commelinaceae & 5 & 8 & Dennstaedtiaceae & 1 & 1 \\
\hline Pteridaceae & 5 & 8 & Erythroxylaceae & 1 & 1 \\
\hline Polypodiaceae & 4 & 7 & Fagaceae & 1 & 1 \\
\hline Scrophulariacae & 7 & 7 & Hernandiaceae & 1 & 1 \\
\hline Agavaceae & 4 & 6 & Hippocrateaceae & 1 & 1 \\
\hline Araceae & 3 & 6 & Iridaceae & 1 & 1 \\
\hline Malpighiaceae & 4 & 6 & Juncaceae & 1 & 1 \\
\hline Sterculiaceae & 4 & 6 & Lauraceae & 1 & 1 \\
\hline Bignoniaceae & 5 & 5 & Loganiaceae & 1 & 1 \\
\hline Celastraceae & 4 & 5 & Loranthaceae & 1 & 1 \\
\hline Crassulaceae & 3 & 5 & Lythraceae & 1 & 1 \\
\hline Cucurbitaceae & 4 & 5 & Marantaceae & 1 & 1 \\
\hline Passifloraceae & 1 & 5 & Martyniaceae & 1 & 1 \\
\hline Piperaceae & 2 & 5 & Menispermaceae & 1 & 1 \\
\hline Myrtaceae & 3 & 4 & Molluginaceae & 1 & 1 \\
\hline Polygonaceae & 4 & 4 & Myrsinaceae & 1 & 1 \\
\hline Portulacaceae & 2 & 4 & Oleaceae & 1 & 1 \\
\hline Rhamnaceae & 4 & 4 & Onagraceae & 1 & 1 \\
\hline Begoniaceae & 1 & 3 & Ophioglossaceae & 1 & 1 \\
\hline Burseraceae & 1 & 3 & Plantaginaceae & 1 & 1 \\
\hline Caryophyllaceae & 1 & 3 & Plumbaginaceae & 1 & 1 \\
\hline Clusiaceae & 1 & 3 & Polygalaceae & 1 & 1 \\
\hline Flacourtiaceae & 2 & 3 & Sapotaceae & 1 & 1 \\
\hline Meliaceae & 2 & 3 & Simarubaceae & 1 & 1 \\
\hline Nyctaginaceae & 2 & 3 & Teophrastaceae & 1 & 1 \\
\hline Oxalidaceae & 1 & 3 & Turneraceae & 1 & 1 \\
\hline Selaginellaceae & 1 & 3 & Violaceae & 1 & 1 \\
\hline Ulmaceae & 2 & 3 & Viscaceae & 1 & 1 \\
\hline \multirow[t]{2}{*}{ Bombacaceae } & 2 & 2 & Zygophyllaceae & 1 & 1 \\
\hline & & & Totales: & 394 & 666 \\
\hline
\end{tabular}




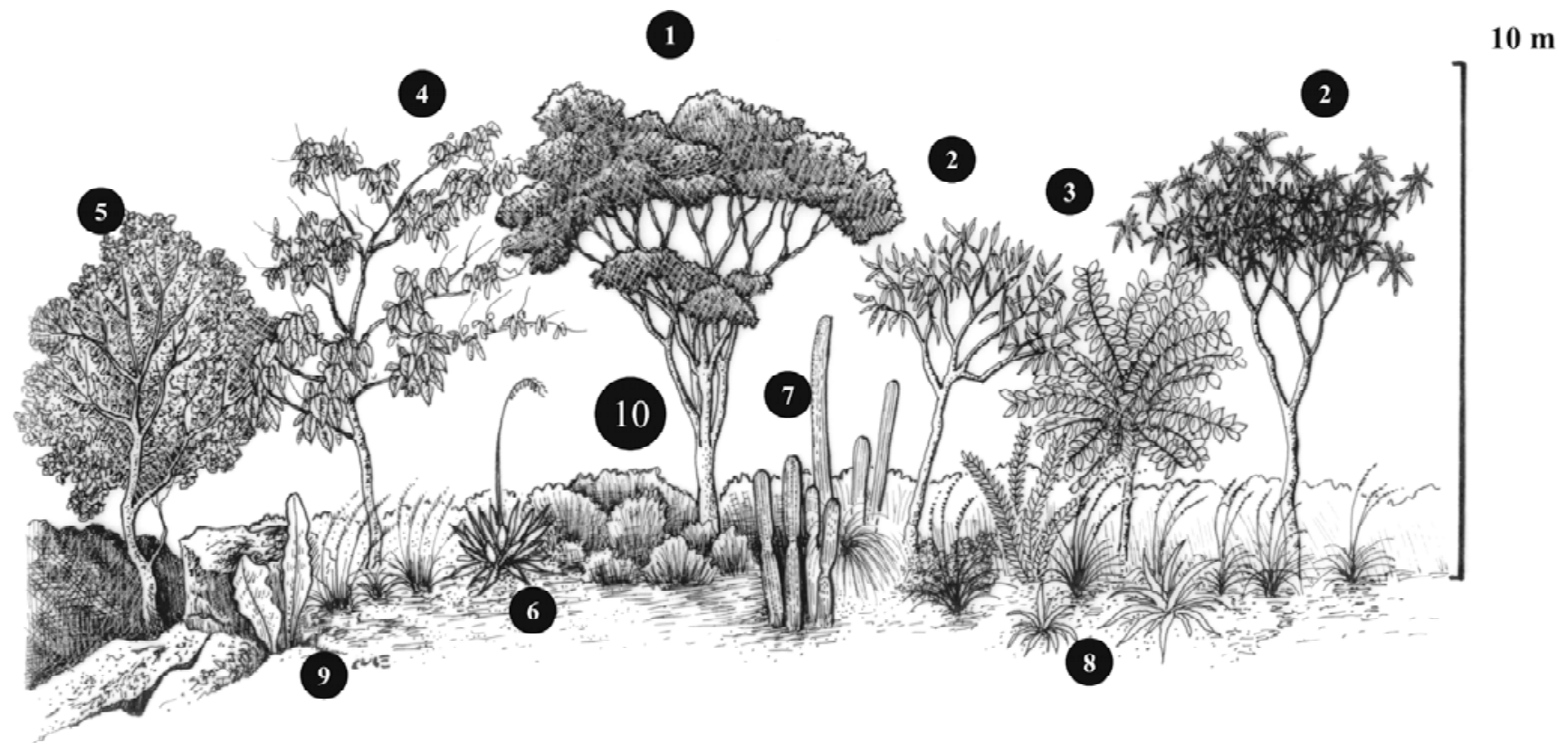

Figura 5. Perfil esquemático de la selva baja caducifolia. 1. Lysiloma acapulcense, 2. Plumeria rubra, 3. Comocladia engleriana, 4. Cochlospermun vitifolium, 5. Bursera cinerea, 6. Agave pendula, 7. Cephalocereus palmeri var. sartorianus, 8. Hechtia sp., 9. Anthurium schlechtendalii subsp. schlechtendalii, 10. Rhynchelytrum repens.

recientes con apenas un año de edad (figura 8a) hasta las etapas avanzadas, con 12-15 años de abandono (figura 8b, c). Esta diferenciación de los estadios de regeneración de la vegetación provoca variabilidad de la diversidad florística, incluyendo cambios de las formas de crecimiento entre los distintos estadios de regeneración: se presentó una disminución de la diversidad de hierbas, hasta casi invertirse con respecto a la diversidad de árboles y arbustos de la vegetación prístina (cuadro 2; figuras 8a, b, c, d). Asimismo, sólo en los estadios más regenerados de la vegetación secundaria (acahual viejo) se observaron epífitas (cuadro 2; figura $8 b, c, d)$.

La estructura de la vegetación secundaria con más de 10 años de abandono presentó de uno a tres estratos: (1) el arbóreo, de 3-7 m de altura, en el que las especies más características fueron Acacia pennatula, Cochlospermum vitifolium, Guazuma ulmifolia, Heliocarps americanus, H. pallidus y Ure racaracasana; (2) el arbustivo, de 1-3 m de alto, caracterizado por Acacia comigera, Calea urtici folia, Calliandra mescens, Cracca caribaea, Croton ciliato-glandulosus, Croton niveus y Pisonia aculeat a; y (3) el herbáceo, de 1-2 m de alto, conformado por Acalypha alopecuroides, Baltimora recta, Bidens pilosa, Desmodium tortuosum, Panicum maximum, Rhynchelytrum repens, Sida rhombifolia y Wissadula amplissima, entre otras.

Es notable la alta riqueza de especies y subespecies que presentó la vegetación secundari a, ya que en esta comunidad se presentó más de la mitad del total de especies y subespecies registradas en la zona de estudio, en comparación con la
Cuadro 2. Síntesis de la diversidad florística por formas de crecimiento de la selva baja caducifolia y de los estadios de regeneración de la vegetación secundaria en áreas de 1,000 m².

\begin{tabular}{lcccc}
\hline & $\begin{array}{c}\text { Acahual } \\
\text { reciente }\end{array}$ & $\begin{array}{c}\text { Acahual } \\
\text { joven }\end{array}$ & $\begin{array}{c}\text { Acahual } \\
\text { viejo }\end{array}$ & $\begin{array}{c}\text { Selva baja } \\
\text { caducifolia }\end{array}$ \\
\hline Árboles & 14 & 26 & 36 & 26 \\
Arbustos & 26 & 54 & 56 & 35 \\
Hierbas & 125 & 106 & 87 & 37 \\
Bejucos & 12 & 20 & 18 & 13 \\
Epífitas & 0 & 1 & 3 & 6 \\
Totales & 177 & 207 & 200 & 117 \\
\hline
\end{tabular}

selva baja caducifolia o vegetación primaria, la cual ocupó una mayor superficie (figuras 4, 6 y 7).

Vegetación ribereña. La vegetación ribereña ocupó una superficie pequeña (26.26 ha). Esta comunidad se concentra principalmente en la planicie fluvial baja y en el lecho del río Naolinco, y limita con la SBC que llega a los márgenes del mismo. Este tipo de vegetación no fue delimitado cartográficamente ni tampoco fue muestreado, porque el valle del río es muy angosto y la SBC y la vegetación secundaria llegan a sus márgenes. Sin embargo, es importante indicarlo porque en los recorridos de campo se detectaron algunos elementos arbóreos y arbustivos propios de la vegetación ribereña. Entre las especies arbóreas más características están Astianthus viminalis, Inga sp. y Salix hum boldtiana. 


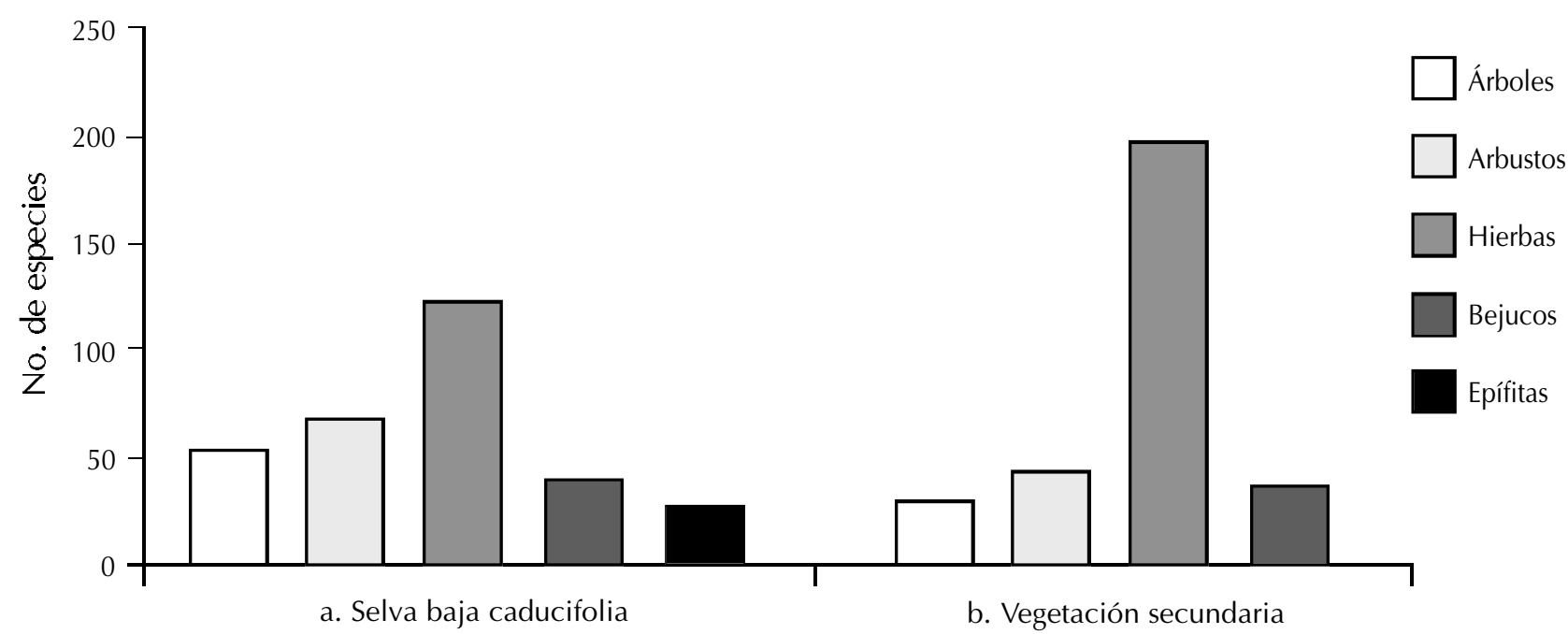

Figura 6. Comparación de la diversidad florística por forma de crecimiento de la vegetación primaria y secundaria.

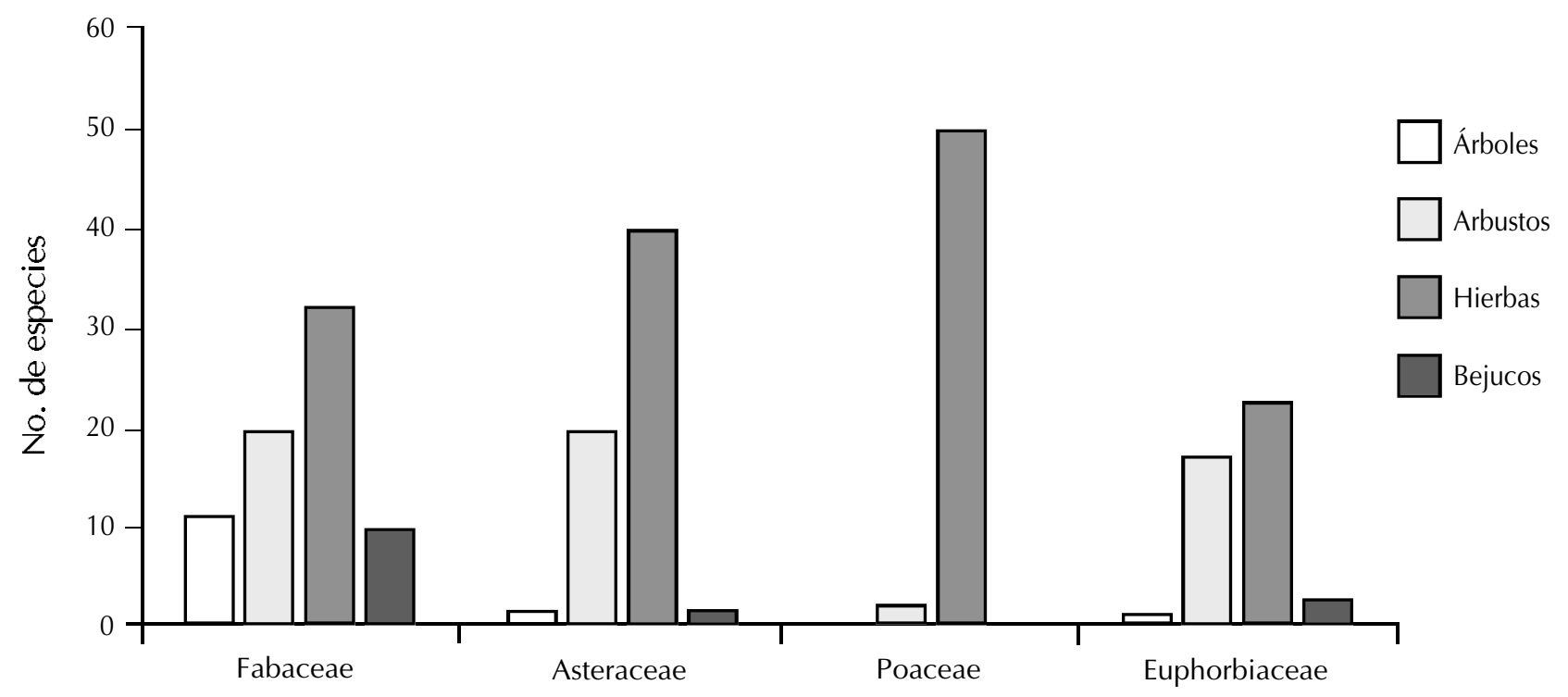

Figura 7. Formas de crecimiento de las familias más diversas de la zona estudiada.

Cultivos. Más de la mitad (55\%) del área correspondió a cultivos y pastizales introducidos e inducidos, los cuales ocuparon 2,228.31 ha, principalmente en las áreas con menos rocosidad y con cenizas volcánicas de espesor variable (figura 4). En las zonas de cultivo no se hicieron muestreos florísticos, con excepción del pastizal. El cultivo de caña de azúcar (Saccharum officinarum), establecido sobre cenizas volcánicas de espesores variables, estuvo bien representado, con una superficie de 1,204.08 ha (figura 4). El pastizal abarcó una superficie de 730.94 ha (figura 4) y se distribuyó en áreas de derrame de basáltico, donde la rocosidad es moderada y existe un recubrimiento delgado y discontinuo de cenizas volcánicas. El pastizal estuvo caracterizado por especies tanto nativas como introducidas, entre las cuales destacaron Homolepis glutinosa, Panicum ghies breghtii, Panicum maximum, Rhynchelytrum repens y Urochloa plantaginea. Las huertas de mango (Mangifera indica) ocuparon una superficie de 211.66 ha, por lo que también fueron importantes en la zona de estudio, y estuvieron asociadas a otros cultivos como el café y el pastizal (figura 4). Por lo general, los cultivos de mango estuvieron ubicados sobre el derrame de basalto, en áreas con escasa rocosidad y pedregosidad inferior a $20 \%$, con topografía plana y recubrimiento continuo y moderadamente espeso de cenizas volcánicas.

El de ch ayote (Sechium edule) fue uno de los dos cultivos que ocupan menor superficie (73.53 ha). Estuvo localizado en la zona más cálida, en altitudes que van de 400 a 

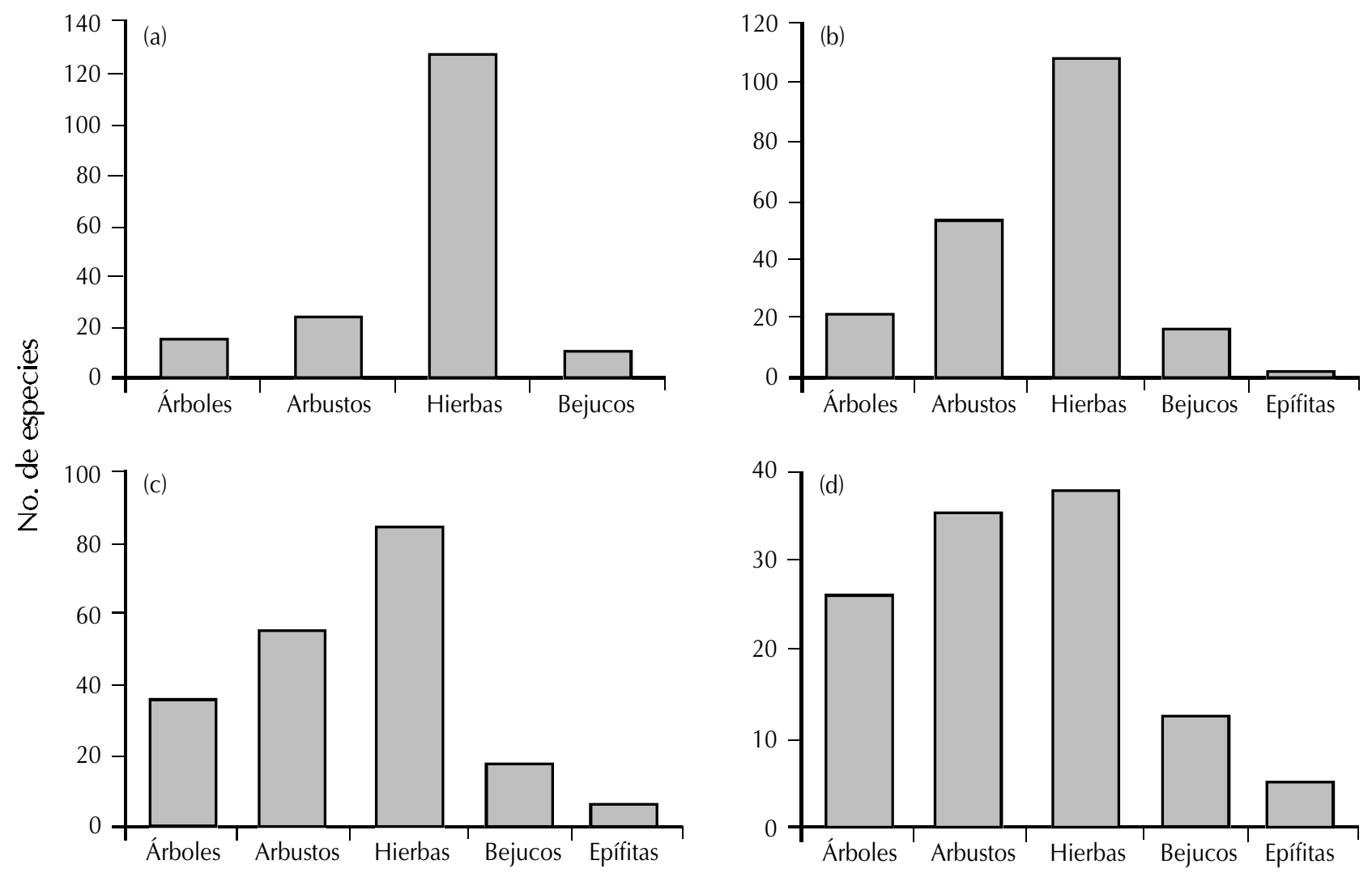

Figuras 8. Comparación de la diversidad florística por forma de crecimiento de la vegetación primaria y de los estadios de regeneración de la vegetación secundaria. (a) acahual reciente; (b) acahual joven; (c) acahual viejo; (d) selva baja caducifolia.

$500 \mathrm{~m}$ (figura 4), y se encontró principalmente sobre el derrame de basalto con rocosidad moderada y topografía lige ramente ondulada, con recubrimiento delgado y discontinuo de cenizas. Por su parte, el cultivo de plátano (Musa sp.) ocupó la superficie más pequeña del área de estudio, con un total de 8.10 ha. Se encontró a $650 \mathrm{~m}$ de altitud, en á reas de escasa rocosidad y una pedregosidad infe rior a $20 \%$, con una topografía plana y recubrimiento continuo y moderadamente profundo de cenizas volcánicas.

\section{Discusión}

Los ambientes rocosos de las zonas tropicales han sido poco estudiados en México; sin embargo, son áreas muy importantes desde el punto de vista ecológico (Meirelles et al., 1999), considerado que en ellas se concentra una alta dive rsidad florística (Castillo-Campos, 2003). En general, se trata de ambientes muy heterogéneos, en los que se localiza un porcentaje importante de la flora endémica (Chiarucci, 1994; Porembski et al., 1994; Gröger y Barthlott, 1996; Silva et al., 1996; Danin, 1999; Meirelles et al., 1999; Castillo-Campos et al., 2005), además de que tienen una baja capacidad para mantener sistemas productivos, por lo que se convierten en áreas potencialmente muy ade- cuadas para la conservación biológica.

La dificultad de conversión de un bosque o una selva a un pastizal o área de cultivo pudo ser constatada en el muestreo de los acahuales de la zona estudiada, donde se encontraron áreas entre los acahuales que escaparon a una perturbación drástica, principalmente en los lugares inaccesibles y con mayor pedregosidad. En ellos, se observó una alta frecuencia de especies suculentas, las cuales están consideradas entre las más sensibles a la perturbación antrópica (figura 5).

La riqueza de la flora de esta zona estuvo caracterizada principalmente por especies herbáceas, las cuales superaron en 50\% a los árboles y arbustos registrados en la zona de estudio (fi g u ra 6a, b). Este hecho se puede ver en las formas de crecimiento (figuras 3 y 6a, b), así como en las familias más diversas como Fabaceae, Asteraceae, Poaceae y Euphorbiaceae (figura 7; cuadro 1), para las que se observ ó que la dive rsidad del estrato herbáceo superó al arbustivo y arbóreo. La antropización de estas comunidades y las limitaciones impuestas por el sustrato rocoso han ocasionado que el estrato herbáceo se vea favorecido, in c rementando la dive rsidad en los dos tipos de vegetación (figura 6a, b).

Contar todavía con una cubierta vegetal original (figura 5) equivalente a $34 \%$ de la SBC en la zona de estudio es 
relevante, considerando que para el estado de Veracruz sólo queda aproximadamente $15 \%$ de cobertura de vegetación original (Guzmán y Castillo, 1989), incluyendo todos los tipos de vegetación registrados para el estado, lo que significa que probablemente hay varios tipos de vegetación que están por desaparecer de Veracruz.

La SBC que crece sobre el sustrato rocoso volcánico es uno de los tipos de vegetación más diversos y donde se concentra un alto porcentaje del endemismo de la flora vascular (Castillo-Campos et al., 2005). Una característica que es necesario hacer notar, y que consideramos importante para la SBC, es la presencia conspicua de una flora suculenta (figura 5). Este resultado es semejante al encontrado en ambientes rocosos calizos por Pérez-García y Meave (2004) en Nizanda, Oaxaca, en el sur de México. Los sustratos rocosos son los menos aptos para el desarrollo de actividades agrícolas, y quizá por ello en el estado de Veracruz no sólo son los que conservan los mejores fragmentos de vegetación original de selva mediana perennifolia (Castillo-Campos et al., 2003) y SBC, sino que probablemente son los que tienen las mejores posibilidades de conservación. Sin embargo, por la inaccesibilidad de las áreas donde están ubicados, también son los menos estudiados. Otro aspecto importante que vale la pena resaltar, es que la SBC establecida en sustratos de este tipo parece ser igual o más diversa que la que se encontraba en mejores condiciones de suelos; desde luego, es difícil constatar esta afirmación, porque esta última ha sido modificada o sustituida en su totalidad por áreas que están destinadas a actividades agropecuarias.

Es notable que la alta diversidad de especies de la flora vascular de la SBC está concentrada en muy pocas familias, a la vez que existe un gran número de familias con pocas especies (cuadro 1). Este patrón es similar a lo rep o rtado por Amiaga y León (1989) para el mismo tipo de vegetación en Baja Califo rnia Sur, México. Como parte de la alta diversidad en los sustratos rocosos volcánicos o malpaís, tanto de la vegetación original como de la secundaria, destacan por su riqueza algunas familias típicas de ambientes antropizados; éste es el caso de Fabaceae, Asteraceae, Poaceae y Euphorbiaceae (figura 7; GómezPompa, 1971). La notable riqueza que caracteriza a la vegetación de los sustratos rocosos se concentra en superficies relativamente pequeñas, ya que en una superficie de 1.2 ha se registraron cerca de 700 especies y subespecies en ambos tipos de vegetación.

La diversidad de la flora leñosa registrada en las familias Bombacaceae, Boraginaceae, Euphorbiaceae, Fabaceae y Rubiaceae es semejante a la citada para Chamela (Durán et al., 2006). Sin embargo, debido a las diferencias de los métodos de muestreo utilizados en Chamela y en este estudio es difícil precisar la diferencia de la diversidad de la flora leñosa de la SBC en las dos localidades.

\section{Agradecimientos}

Agradecemos a Luciana Porter por la traducción del resumen al inglés. Alejandro Flores Palacios, José Antonio Vázquez García, Óscar Dorado y Sergio Avendaño Reyes revisaron el manuscrito, y Klaus Mehltreter hizo la revisión nomenclatural de las Pteridofitas. Este trabajo se realizó con financiamiento del Instituto de Ecología, A.C., a través del Departamento de Biodiversidad y Sistemática (902-14) y formó parte de la tesis doctoral del primer autor (Programa de Posgrado en Ciencias Biológicas, Universidad Autónoma Metropolitana-Iztapalapa). Nuestro sincero agradecimiento a Guadalupe Rivera Vega por su apoyo en dar formato al manuscrito, a Braian Sánchez González, Pablo O. Aguilar y María Elena Medina por su apoyo técnico, a Israel Acosta Rosado por su apoyo técnico en el campo, a Manuel Escamilla por la elaboración del perfil de la vegetación y a Lamberto Aragón por su apoyo en informática.

\section{Literatura citada}

Acosta P.R. 1986. La vegetación de la Sierra de Manuel Díaz, Veracruz, México. Tesis de Licenciatura, Facultad de Biología, Universidad Veracruzana, Xalapa, 96 pp.

Arriaga L. y León J.L. 1989. The Mexican tropical deciduous forest of Baja California Sur: a floristic and structural approach. Plant Ecology 84:45-52.

Brummitt R.K. y Powell C.E. Eds. 1992. Authors of Plant Names. A List of Authors of Scientific Names of Plants, with Recommended Standard Form of Their Names, Including Abbreviations. Royal Botanic Gardens, Kew.

Castillo-Campos G. 1985. Integración de paisajes en la región de Jalcomulco, Veracruz. Tesis de Licenciatura, Facultad de Biología, Universidad Veracruzana, Xalapa, 110 pp.

Castillo-Campos G. 1995. Ecología del paisaje del municipio de Jalcomulco, Veracruz. Tesis de Maestría, Facultad de Ciencias, Universidad Nacional Autónoma de México, México, D.F., $192 \mathrm{pp}$.

Castillo-Campos G. 2003. Biodiversidad de la selva baja caducifolia en un sustrato rocoso de origen volcánico en el centro del estado de Veracruz, México. Tesis de Doctorado, Universidad Autónoma Metropolitana-Iztapalapa, México, D.F., 204 pp.

Castillo-Campos G., Medina-Abreo M.E., Dávila-Aranda P.D. y Zavala-Hurtado J.A. 2005. Contribución al conocimiento del endemismo de la flora vascular en Veracruz, México. Acta Botanica Mexicana 73:19-57.

Castillo-Campos G., Robles G.R. y Medina-Abreo M.E. 2003. Flora y vegetación de la sierra Cruz Tetela, Veracruz, México. Polibotánica 15:41-87.

Castillo-Campos G., Vovides A. y Avendaño S. 1999. Riqueza y diversidad de los sustratos rocosos del centro del estado de Veracruz (Informe Final a CONABIO, inédito). 120 pp.

Castillo-Campos G., Vovides A. y Vázquez M. 1998. Una nueva especie de Stromanthe (Marantaceae) de Veracruz, México. Polibotánica 8:13-19.

Cházaro M. de J. 1992. Exploraciones botánicas en Veracruz y estados circunvecinos I. Pisos altitudinales de vegetación en el 
centro de Veracruz y zonas limítrofes con Puebla. La Ciencia y el Hombre 10:67-115.

Chiarucci A. 1994. Successional pathway of Mediterranean ultramafic vegetation in central Italy. Acta Botanica Croatica 53:93-94.

Cronquist A. 1988. The Evolution and Classification of Flowering Plants. The New York Botanical Garden, Nueva York.

Danin A. 1999. Sandstone outcrops: a major refugium of Mediterranean flora in the xeric part of Jordan. Israel Journal of Plant Sciences 47:179-187.

Durán E., Meave J.A., Lott E.J. y Segura G. 2006. Structure and tree diversity patterns at the landscape level in a Mexican tropical deciduous forest. Boletín de la Sociedad Botánica de México 79:43-60.

García E. 1988. Modificaciones al Sistema de Clasificación Climática de Köppen (Para Adaptarlo a las Condiciones de la República Mexicana). Editado por la autora. 4a. ed. México, D.F.

Gómez-Pompa A. 1971. Posible papel de la vegetación secundaria en la evolución de la flora tropical. Biotropica 3:125-135.

Gómez-Pompa A. 1978. Ecología de la Vegetación del Estado de Veracruz. Instituto Nacional de Investigaciones Sobre Recursos Bióticos y CECSA, México, D.F.

Gómez-Pompa A., Vovides A., Ogata N. y González J. 1994. Las cícadas de México. CD-ROM interactivo. Gestión de Ecosistemas, México, D.F.

Gröger A. y Barthlott W. 1996. Biogeography and diversity of the inselberg (laja) vegetation of southern Venezuela. Biodiversity Letters 3:165-179.

Guzmán G.S. y Castillo-Campos G. 1989. Uso del suelo en Veracruz. Extensión 32:31-35.

INEGI [Instituto Nacional de Estadística, Geografía e Informática]. 1987a. Carta Estatal de Hidrología Superficial del Estado de Veracruz, escala 1:1,000,000. México, D.F.

INEGI. 1987b. Carta Estatal de Climas del Estado de Veracruz, escala 1:1,000,000. México, D.F.

Lott, E.J., Bullock S.H. y Solís-Magallanes. J.A. 1987. Floristic diversity and structure of upland and arroyo forest of coastal Jalisco. Biotropica 19:228-235.

Medina-Abreo M.E. y Castillo-Campos G. 1993. Vegetación y listado florístico de la barranca de Acazónica, Veracruz. Boletín de la Sociedad Botánica de México 53:73-111.

Medina-Abreo M.E. y Castillo-Campos G. 1996. Las plantas suculentas de la barranca de Acazónica, Ver. Cactáceas y Suculentas Mexicanas 41:12-17.

Meirelles S.T., Pivello V.R. y Oly C.A. 1999. The vegetation of granite rock outcrops in Rio de Janeiro, Brasil, and the need for its protection. Environmental Conservation 26:10-20.

Miranda F. y Hernández-X E. 1963. Los tipos de vegetación de
México y su clasificación. Boletín de la Sociedad Botánica de México 28:29-72.

Narave F.H. 1985. La vegetación del Cofre de Perote, Veracruz. México. Biotica 10:35-64.

Negendank J.F., Emmermann W.R., Krawczyk R., Mooser F., Tobschall H. y Werle D. 1985. Geological and geochemical investigations on the Eastern Trans-Mexican Belt. Geofísica Internacional 24:477-575.

Ortega O.R. 1981. Vegetación y flora de una corriente de lava (malpaís) al noreste del Cofre de Perote, Veracruz. Biotica 6:57-97.

Pérez-García E.A. y Meave J.A. 2004. Heterogeneity of xerophytic vegetation of limestone outcrops in a tropical deciduous forest region in southern Mexico. Plant Ecology 175:147-163.

Porembski S., Barthlott W., Dörrstock S. y Biedinger N. 1994. Vegetation of rock outcrops in Guinea: granite inselbergs, sandstone table mountains and ferricretes - remarks on species numbers and endemism. Flora 189:315-326.

Rossignol J. P. 1987. Morfoedafología del Área XalapaCoatepec: Capacidad de Uso Agrícola, Pecuario y Forestal, escala 1:75,000. Instituto Nacional de Investigaciones Sobre Recursos Bióticos y Institut de Recherche pour le Développement (ORSTOM), Xalapa.

Rossignol J. P. y Geissert D. 1987. Morfoedafología del Área Xalapa-Coatepec: Recursos en Tierras, escala 1:75,000. Instituto Nacional de Investigaciones Sobre Recursos Bióticos y Institut de Recherche pour le Développement (ORSTOM), Xalapa.

Rzedowski J. 1978. Vegetación de México. Limusa, México, D.F. Rzedowski J. 1991. Diversidad y orígenes de la flora fanerogámica de México. Acta Botanica Mexicana 14:3-21.

Rzedowski J. y Calderón de R. G. 1987. El bosque tropical caducifolio de la región mexicana del Bajío. Trace 12:12-21.

Sarukhán J. 1998. Los tipos de vegetación arbórea de la zona cálida húmeda de México. En: Pennington T.D. y Sarukhán J. Eds. Árboles Tropicales de México, pp. 13-65, Universidad Nacional Autónoma de México y Fondo de Cultura Económica, México, D.F.

Silva M.F.F., Secco R.S. y Lobo M.G.A. 1996. Ecological aspects of the tropical scrub vegetation on rocky outcrops of the Serra dos Carajas, state of Para, Brazil. Acta Amazonica 26:17-44.

Toledo V.M. 1982. Pleistocene changes of vegetation in tropical México. En: Prance G.T. Ed. Biological Diversification in the Tropics, pp. 93-111, Columbia University Press, Nueva York.

$\mathrm{W}^{3}$ Tropicos. Missouri Botanical Garden's VAST nomenclatural database and associated authority files. <mobot.mobot.org/W3T/Search/vast.html> (consultada durante 2004)

Recibido: 18 de marzo de 2005

Versión corregida: 12 de marzo de 2007

Aceptado: 9 de abril de 2007 
Apéndice 1. Lista florística de la vegetación de un derrame de lava en el centro de Veracruz, México. Se han incorporado las especies nativas, así como algunas cultivadas, registradas en los muestreos realizados en la selva baja caducifolia del malpaís que comprende el área de estudio. También se diferencian las especies nativas de las cultivadas, las endémicas de México y de Veracruz, la forma biológica y el tipo de comunidad a la que pertenecen (original o antropizada). (XAL) = Herbario XAL del Instituto de Ecología, A.C. $(\mathbf{C})=$ cultivada, $(\mathbf{E M})=$ endémica de México, $(\mathbf{E V})=$ endémica de Veracruz, $(\mathbf{A})=$ árbol, $(\mathbf{A r})=$ arbusto, $(\mathbf{H})=$ hierba, $(\mathbf{B})=$ bejuco, $(\mathbf{E})=$ epífita, $(\mathbf{P})=$ vegetación original, $(\mathbf{S})$ = vegetación secundaria.

\begin{tabular}{|c|c|c|c|c|c|c|c|c|c|c|}
\hline Familia y especie & C & EM & EV & A & $\operatorname{Ar}$ & $\mathrm{H}$ & B & $\mathrm{E}$ & $\mathrm{P}$ & $\mathrm{S}$ \\
\hline \multicolumn{11}{|l|}{ PTERIDOPHYTA Y GRUPOS AFINES } \\
\hline \multicolumn{11}{|l|}{ ASPLENIACEAE } \\
\hline Asplenium pumilum Sw. (XAL) 18333 & - & - & - & - & - & $x$ & - & - & $X$ & - \\
\hline DENNSTAEDTIACEAE & - & - & - & - & - & $X$ & - & - & $\mathrm{X}$ & - \\
\hline \multicolumn{11}{|l|}{ Pteridium arachnoideum (Kaulf.) Maxon (XAL) 17152} \\
\hline \multicolumn{11}{|l|}{ Ophioglossum reticulatum L. (XAL) 19951} \\
\hline POLYPODIACEAE & - & - & - & - & - & $X$ & - & - & $X$ & - \\
\hline Microgramma nitida (J.Sm.) A.R.Sm. (XAL) 16621 & - & - & - & - & - & $X$ & - & - & $\mathrm{X}$ & - \\
\hline Pecluma atra (A.M.Evans) M.G.Price (XAL) 19458 & - & - & - & - & - & $X$ & - & - & $X$ & - \\
\hline \multicolumn{11}{|l|}{ Pecluma plumula (Humb. et Bonpl. ex Willd.) } \\
\hline M.G.Price (XAL) 17281 & - & - & - & - & - & $X$ & - & - & $X$ & - \\
\hline \multicolumn{11}{|l|}{ Phlebodium areolatum (Humb. et Bonpl. ex Willd.) } \\
\hline J.Sm. (XAL) 17504 & - & - & - & - & - & $X$ & - & - & $X$ & - \\
\hline Polypodium plesiosorum Kunze (XAL) 18466 & - & - & - & - & - & $x$ & - & - & $X$ & - \\
\hline \multicolumn{11}{|l|}{ Polypodium polypodioides (L.) Watt. var. aciculare } \\
\hline Weath. (XAL) 18529 & - & - & - & - & - & $x$ & - & - & $X$ & - \\
\hline \multicolumn{11}{|l|}{ Polypodium thyssanolepis A.Braun ex Klotzsch (XAL) } \\
\hline 18458 & - & - & - & - & - & $x$ & - & - & $X$ & - \\
\hline \multicolumn{11}{|l|}{ PTERIDACEAE } \\
\hline \multicolumn{11}{|l|}{ Astrolepis sinuata (Lag. ex. Sw.) D.M.Benham et } \\
\hline Windham subsp. sinuata (XAL) 16620 & - & - & - & - & - & $x$ & - & - & $X$ & - \\
\hline Cheilanthes bonariensis (Willd.) Proctor (XAL) 16366 & - & - & - & - & - & $x$ & - & - & $X$ & - \\
\hline Cheilanthes eatonii Baker (XAL) 19100 & - & - & - & - & - & $x$ & - & - & $X$ & - \\
\hline Cheilanthes kaulfussii Kunze (XAL) 19223 & - & - & - & & - & $x$ & - & - & $X$ & - \\
\hline Cheilanthes microphylla (Sw.) Sw. (XAL) 18582 & - & - & - & - & - & $x$ & - & - & $X$ & - \\
\hline Hemionitis palmata L. (XAL) 19436 & - & - & - & - & - & $x$ & - & - & $X$ & - \\
\hline \multicolumn{11}{|l|}{ Mildella intramarginalis (Kaulf. ex Link) Trevis. } \\
\hline (XAL) 17292 & - & - & - & - & - & $x$ & - & - & $X$ & - \\
\hline Pellaea ternifolia (Cav.) Link & - & - & - & - & - & $X$ & - & - & $X$ & - \\
\hline \multicolumn{11}{|l|}{ SCHIZAEACEAE } \\
\hline Anemia karwinskyana (C.Presl) Prantl (XAL) & - & - & - & - & - & $x$ & - & - & $X$ & - \\
\hline \multicolumn{11}{|l|}{ Anemia tomentosa (Savigny) Sw. var. mexicana } \\
\hline (C.Presl) Mickel (XAL) 16619 & - & - & - & - & - & $x$ & - & - & $X$ & - \\
\hline \multicolumn{11}{|l|}{ SELAGINELLACEAE } \\
\hline Selaginella pallescens (C.Presl) Spring (XAL) 17333 & - & - & - & - & - & $X$ & - & - & $\mathrm{X}$ & - \\
\hline Selaginella sartorii Hieron. (XAL) 17503 & - & - & - & - & - & $X$ & - & - & $X$ & - \\
\hline Selaginella schiedeana A.Braun (XAL) 19470 & - & - & - & - & - & $x$ & - & - & $\mathrm{X}$ & - \\
\hline \multicolumn{11}{|l|}{ MONOCOTILEDÓNEAS } \\
\hline \multicolumn{11}{|l|}{ AGAVACEAE } \\
\hline \multicolumn{11}{|l|}{ Agave angustifolia Haw. var. angustifolia (XAL) } \\
\hline 16652 & - & $\mathrm{X}$ & - & - & - & $X$ & - & - & $X$ & - \\
\hline Agave pendula Schnittsp. (XAL) 17883 & - & 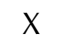 & - & - & - & $X$ & - & - & $X$ & - \\
\hline Agave obscura Schiede (XAL) 18615 & - & - & - & - & - & $X$ & - & - & $x$ & - \\
\hline Beaucarnea recurvata Lem. (XAL) 18319 & - & - & - & $X$ & - & - & - & - & $X$ & - \\
\hline Manfreda scabra (Ortega) McVaugh (XAL) 18480 & - & - & - & - & - & $X$ & - & - & $X$ & - \\
\hline Yucca elephantipes Regel (XAL) 17988 & $x$ & - & - & $x$ & - & - & - & - & $X$ & - \\
\hline
\end{tabular}




$\begin{array}{lllllll}A & \text { Ar } & H & \text { B } & \text { E } & P & S\end{array}$

\section{ARACEAE}

Anthurium scandens (Aubl.) Engl. subsp. scandens (XAL)

Anthurium schlechtendalii Kunth subsp. schlechtendalii (XAL) 19385

Philodendron hederaceum (Jacq.) Schott (XAL) 19517

Philodendron inaequilaterum Liebm. (XAL) 19518

Philodendron radiatum Schott (XAL) 17900

Syngonium podophyllum Schott (XAL) 19383

\section{ARECACEAE}

Acrocomia aculeata (Jacq.) Lodd. ex Mart. (XAL)

\section{BROMELIACEAE}

Aechmea bracteata (Sw.) Griseb. (XAL) 17230

Bromelia pinguin L. (XAL) 19036

Catopsis nutans (Sw.) Griseb. (XAL)

Hechtia sp. (XAL) 17058

Hechtia sp. (XAL) 19217

Hechtia stenopetala Klotzsch (XAL) 16651

Tillandsia balbisiana Schult.f. (XAL) 19445

Tillandsia fasciculata Sw. (XAL) 19215

Tillandsia grandis Schltdl. (XAL) 17967

Tillandsia ionantha Planch. (XAL) 19443

Tillandsia juncea (Ruiz et Pav.) Poir. (XAL) 17025

Tillandsia paucifolia Baker (XAL) 16404

Tillandsia recurvata (L.) L. (XAL) 19211

Tillandsia schiedeana Steud. (XAL) 10370

Tillandsia sp. (XAL) 17072

Tillandsia usneoides (L.) L. (XAL) 17185

\section{COMMELINACEAE}

Callisia fragrans (Lindl.) Woodson (XAL) 18346

Callisia multiflora (M.Martens et Galeotti) Standl.

(XAL) 17235

Callisia repens (Jacq.) L. (XAL) 16625

Commelina erecta L. (XAL) 18158

Gibasis pellucida (M.Martens et Galeotti) D.R.Hunt (XAL) 19515

Tinantia erecta (Jacq.) Schltdl. (XAL) 18434

Tradescantia commelinoides Schult. et Schult.f. (XAL) 19414

Tradescantia zanonia (L.) Sw. (XAL) 17520

\section{CYPERACEAE}

Bulbostylis capillaris (L.) C.B.Clarke (XAL) 19203

Cyperus aggregatus (Willd.) Endl. (XAL) 18117

Cyperus hermaphroditus (Jacq.) Standl. (XAL) 19368

Cyperus mutisii (Kunth) Griseb. var. mutisii (XAL) 19428

Cyperus rotundus L. (XAL)

Cyperus surinamensis Rottb. (XAL)

Cyperus tenuis Sw. (XAL)

Fimbristylis autumnalis (L.) Roem. et Schult. (XAL) 19040

Kyllinga brevifolia Rottb. (XAL)

\section{DIOSCOREACEAE}

Dioscorea convolvulacea Schltdl. et Cham. (XAL) 18420 


\begin{tabular}{|c|c|c|c|c|c|c|c|c|c|c|}
\hline Familia y especie & $\mathrm{C}$ & EM & EV & A & $\operatorname{Ar}$ & $\mathrm{H}$ & B & $\mathrm{E}$ & $\mathrm{P}$ & $\mathrm{S}$ \\
\hline Dioscorea floribunda M.Martens et Galeotti (XAL) & - & - & - & - & - & - & $x$ & - & $x$ & - \\
\hline \multicolumn{11}{|l|}{ 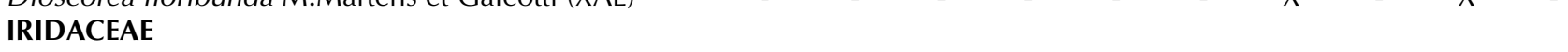 } \\
\hline \multicolumn{11}{|l|}{ Eleutherine latifolia (Standl. et L.O.Williams) Ravenna } \\
\hline \multicolumn{11}{|l|}{ JUNCACEAE } \\
\hline Juncus sp. (XAL) & - & - & - & - & - & $x$ & - & - & - & $x$ \\
\hline \multicolumn{11}{|l|}{ MARANTACEAE } \\
\hline Maranta arundinacea L. (XAL) 18256 & - & - & - & - & - & $\mathrm{x}$ & - & - & - & $\mathrm{x}$ \\
\hline \multicolumn{11}{|l|}{ ORCHIDACEAE } \\
\hline Beloglottis mexicana Garay et Hamer (XAL) 17892 & - & - & - & - & - & - & - & $\mathrm{x}$ & $\mathrm{x}$ & - \\
\hline Cranichis sp. (XAL) 17530 & - & - & - & - & - & - & - & $\mathrm{x}$ & $\mathrm{x}$ & - \\
\hline \multicolumn{11}{|l|}{ Cyrtopodium macrobulbum (La Llave et Lex.) } \\
\hline G.A.Romero et Carnevali (XAL) 17901 & - & - & - & - & - & - & - & $x$ & $x$ & - \\
\hline Encyclia parviflora (Regel) Wither & - & - & - & - & - & - & - & $\mathrm{x}$ & $\mathrm{x}$ & - \\
\hline Govenia utriculata (Sw.) Lindl. (XAL) 18134 & - & - & - & - & - & $\mathrm{x}$ & - & - & $\mathrm{X}$ & - \\
\hline \multicolumn{11}{|l|}{ Lycaste aromatica (Graham ex Hook.) Lindl. (XAL) } \\
\hline 17217 & - & - & - & - & - & - & - & $\mathrm{x}$ & $\mathrm{x}$ & - \\
\hline \multicolumn{11}{|l|}{ Myrmecophila grandiflora (Lindl.) Carnevali, } \\
\hline Tapia-Muñoz et I.Ramírez (XAL) 17031 & - & - & - & - & - & - & _. & $\mathrm{x}$ & $x$ & - \\
\hline Ponthieva racemosa (Walter) C.Mohr (XAL) 16708 & - & - & - & - & - & - & - & $\mathrm{x}$ & $\mathrm{x}$ & - \\
\hline \multicolumn{11}{|l|}{ Prosthechea cochleata (L.) W.E.Higgings (XAL) } \\
\hline 16997 & - & - & - & - & - & - & - & $\mathrm{x}$ & $\mathrm{X}$ & - \\
\hline Prosthechea livida (Lindl.) W.E.Higgins (XAL) 17221 & - & - & - & - & - & - & - & $\mathrm{x}$ & $\mathrm{x}$ & - \\
\hline Sarcoglottis schaffneri (Rchb.f.) Ames (XAL) 19468 & - & - & - & - & - & - & - & $\hat{x}$ & $x$ & - \\
\hline \multicolumn{11}{|l|}{ Scaphyglottis lindeniana (A.Rich. et Galeotti) } \\
\hline \multirow{2}{*}{\multicolumn{11}{|c|}{ Specklinia tribuloides (Sw.) Pridgeon et M.W.Chase }} \\
\hline & & & & & & & & & & \\
\hline$(\mathrm{XAL})$ & - & - & - & - & - & - & - & $x$ & $x$ & - \\
\hline \multicolumn{11}{|l|}{ Trichocentrum cosymbephorum (C.Morren) R.Jiménez } \\
\hline et Carnevali (XAL) 19369 & - & - & - & - & - & $x$ & _ & - & $\mathrm{x}$ & - \\
\hline \multicolumn{11}{|l|}{ POACEAE } \\
\hline Anthephora hermaphrodita (L.) Kuntze (XAL) 19296 & - & - & - & - & - & $x$ & _- & - & _ & $x$ \\
\hline Aristida ternipes Cav. var. ternipes (XAL) 16747 & - & - & - & - & - & $\mathrm{x}$ & - & - & - & $x$ \\
\hline Axonopus compressus (Sw.) P.Beauv. (XAL) & $x$ & - & - & - & - & $\mathrm{x}$ & _ & - & _ & $\mathrm{x}$ \\
\hline Axonopus fissifolius (Raddi) Kuhlm. (XAL) & - & - & - & - & - & $\mathrm{x}$ & - & - & - & $x$ \\
\hline \multicolumn{11}{|l|}{ Bouteloua repens (Kunth) Scribn. et Merr. (XAL) } \\
\hline 19481 & - & - & - & - & - & $x$ & - & - & - & $x$ \\
\hline Bouteloua sp. (XAL) 16445 & - & - & - & - & - & $\mathrm{x}$ & - & - & - & $\mathrm{x}$ \\
\hline Cenchrus echinatus L. (XAL) 19345 & - & - & - & - & - & $\mathrm{x}$ & - & - & - & $\mathrm{x}$ \\
\hline Chloris virgata Sw. (XAL) 19297 & - & - & - & - & - & $\mathrm{x}$ & - & - & - & $\mathrm{x}$ \\
\hline Cynodon dactylon (L.) Pers. (XAL) 19329 & $\mathrm{x}$ & - & - & - & - & $\mathrm{x}$ & - & - & - & $\mathrm{x}$ \\
\hline Cynodon plectostachyus (K.Schum.) Pilg. (XAL) & $\mathrm{x}$ & - & - & - & - & $\mathrm{x}$ & - & - & - & $x$ \\
\hline Dactyloctenium aegyptium (L.) Willd. (XAL) 19321 & - & - & - & - & - & $\mathrm{x}$ & _. & - & _ & $\mathrm{x}$ \\
\hline Digitaria bicornis (Lam.) Roem. et Schult. (XAL) & $\mathrm{x}$ & - & - & - & - & $\mathrm{x}$ & - & - & - & $x$ \\
\hline Digitaria ciliaris (Retz.) Koeler (XAL) & - & - & - & - & - & $\mathrm{x}$ & _ & - & _ & $\mathrm{x}$ \\
\hline Digitaria insularis (L.) Fedde (XAL) & - & - & - & - & - & $\hat{x}$ & - & - & - & $x$ \\
\hline Digitaria sp. (XAL) & - & _. & - & - & - & $\mathrm{x}$ & - & - & - & $\mathrm{x}$ \\
\hline Echinochloa colonum (L.) (XAL) & $x$ & - & - & - & - & $x$ & - & - & - & $x$ \\
\hline Eleusine indica (L.) Gaertn. var. indica (XAL) 19500 & $\mathrm{x}$ & - & - & - & - & $\mathrm{x}$ & - & - & - & $\mathrm{x}$ \\
\hline \multicolumn{11}{|l|}{ Eragrostis cilianensis (All.) Vignolo ex Janch. (XAL) } \\
\hline 19029 & - & - & - & - & - & $\mathrm{x}$ & - & - & - & $\mathrm{x}$ \\
\hline Eragrostis ciliaris (L.) R.Br. var. ciliaris (XAL) 19479 & $\mathrm{x}$ & - & - & - & - & $\mathrm{x}$ & - & - & - & $\mathrm{x}$ \\
\hline Eragrostis lugens Nees $(\mathrm{XAL})$ & - & - & - & - & - & $\mathrm{x}$ & - & - & - & $\mathrm{x}$ \\
\hline Eragrostis sp. (XAL) 16838 & - & - & - & - & - & $\mathrm{x}$ & _ & - & _ & $\mathrm{x}$ \\
\hline Eriochloa punctata (L.) Desv. ex Ham. (XAL) & - & - & - & - & - & $x$ & - & - & - & $x$ \\
\hline
\end{tabular}




$\operatorname{Ar}$

Gouinia virgata (J.Presl) Scribn. var. virgata (XAL) 19388

Heteropogon contortus (L.) P.Beauv. ex Roem. et Schult. (XAL) 16981

Homolepis glutinosa (Sw.) Zuloaga et Soderstr. (XAL)

Hyparrhenia rufa (Nees) Stapf (XAL) 17086

Lasiacis aff. ruscifolia (Kunth) Hitchc. (XAL)

Lasiacis aff. sorghoidea (Desv. ex Ham.) Hitchc. Et Chase (XAL)

Lasiacis nigra Davidse (XAL) 18222

Lasiacis rugelii (Griseb.) Hitchc. (XAL) 18133

Lasiacis ruscifolia (Kunth) Hitchc. (XAL)

Leptochloa mucronata (Michx.) Kunth (XAL)

Leptochloa virgata (L.) P.Beauv. (XAL)

Oplismenus burmannii (Retz.) P.Beauv. var. burmannii (XAL)

Oplismenus hirtellus (L.) P.Beauv. subsp. setarius (Lam.) Mez ex Ekman (XAL)

Panicum ghiesbreghtii E.Fourn. (XAL) 17263

Panicum trichanthum Nees (XAL) 17348

Paspalum conjugatum P.J.Bergius var. conjugatum (XAL)

Paspalum langei (E.Fourn.) Nash (XAL)

Paspalum notatum Flüggé (XAL) 18488

Paspalum plicatulum Michx. (XAL)

Paspalum virgatum $\mathrm{L}$. (XAL)

Paspalum sp. (XAL) 2

Paspalum sp. (XAL) 3

Rhynchelytrum repens (Willd.) C.E.Hubb. (XAL) 16623

Setaria macrostachya Kunth (XAL) 18227

Setaria parviflora (Poir.) Kerguélen (XAL)

Setaria aff. grisebachii E.Fourn. (XAL) 18393

Setariopsis auriculata (E.Fourn.) Scribn. (XAL) 18805

Sporobolus indicus (L.) R.Br. (XAL)

Trachypogon plumosus (Humb. et Bonpl. ex Willd.) Nees (XAL) 18461

Urochloa fasciculata (Sw.) R.D.Webster var. fasciculata (XAL)

Urochloa maxima (Jacq.) R.D.Webster (XAL) 19043

Urochloa plantaginea (Link) R.D.Webster (XAL)

Zea mays L. (XAL)

\section{DICOTILEDÓNEAS}

\section{ACANTHACEAE}

Aphelandra deppeana Schltdl. et Cham. (XAL) 16967

Blechum brownei Juss. (XAL)

Dyschoriste quadrangularis (Oerst.) Kuntze (XAL)

Elytraria bromoides Oerst. (XAL) 19415

Elytraria imbricata (Vahl) Pers. (XAL) 18032

Henrya insularis Nees ex Benth. (XAL) 17601

Justicia comata (L.) Lam. (XAL)

Ruellia sp. (XAL) 17562

Siphonoglossa sessilis (Jacq.) D.N.Gibson (XAL)

\begin{tabular}{|c|c|c|c|c|c|c|c|c|c|}
\hline - & - & - & - & - & $X$ & - & - & $x$ & - \\
\hline - & - & - & - & - & $X$ & - & - & $x$ & - \\
\hline - & - & - & - & - & $x$ & - & - & $x$ & - \\
\hline X & - & - & - & - & $X$ & - & - & $x$ & . \\
\hline - & - & - & - & $x$ & - & - & - & $x$ & - \\
\hline- & - & - & - & $x$ & - & - & - & $X$ & - \\
\hline - & - & - & - & - & $X$ & - & - & $X$ & - \\
\hline - & - & - & - & - & $X$ & - & - & $X$ & - \\
\hline - & - & - & - & - & $X$ & - & - & - & $X$ \\
\hline- & - & - & - & - & $X$ & - & - & - & $X$ \\
\hline - & - & - & - & - & $X$ & - & - & - & $X$ \\
\hline - & - & - & - & - & $X$ & - & - & $X$ & - \\
\hline - & - & - & - & - & $X$ & - & - & $X$ & - \\
\hline - & - & - & - & - & $X$ & - & - & - & $X$ \\
\hline - & - & - & - & - & $X$ & - & - & $X$ & - \\
\hline- & - & - & - & - & $X$ & - & - & - & $X$ \\
\hline- & - & - & - & - & $X$ & - & - & $X$ & - \\
\hline- & - & - & - & - & $X$ & - & - & - & $x$ \\
\hline- & - & - & - & - & $x$ & - & - & - & $X$ \\
\hline - & - & - & - & - & $X$ & - & - & - & $X$ \\
\hline - & - & - & - & - & $X$ & - & - & - & $x$ \\
\hline- & - & - & - & - & $X$ & - & - & - & $X$ \\
\hline- & - & - & - & - & $x$ & - & - & $x$ & - \\
\hline - & - & - & - & - & $x$ & - & - & $X$ & - \\
\hline- & - & - & - & - & $x$ & - & - & $x$ & - \\
\hline - & - & - & - & - & $X$ & - & - & $X$ & - \\
\hline - & - & - & - & - & $X$ & - & - & - & $x$ \\
\hline - & - & - & - & - & $X$ & - & - & - & $X$ \\
\hline - & - & - & - & - & $X$ & - & - & $X$ & - \\
\hline - & - & - & - & - & $x$ & - & - & - & $x$ \\
\hline - & - & - & - & - & $X$ & - & - & - & $x$ \\
\hline - & - & - & - & - & $x$ & - & - & - & $x$ \\
\hline - & - & - & - & - & $X$ & - & - & - & $X$ \\
\hline
\end{tabular}




\begin{tabular}{|c|c|c|c|c|c|c|c|c|c|c|}
\hline Familia y especie & $\mathrm{C}$ & EM & EV & A & $\operatorname{Ar}$ & $\mathrm{H}$ & B & $\mathrm{E}$ & $\mathrm{P}$ & $\mathrm{S}$ \\
\hline Tetramerium nervosum Nees (XAL) & - & - & - & - & - & $x$ & - & - & $x$ & - \\
\hline Thunbergia alata Bojer ex Sims (XAL) & $x$ & - & - & - & - & - & $x$ & - & - & $x$ \\
\hline \multicolumn{11}{|l|}{ AMARANTHACEAE } \\
\hline Achyranthes aspera L. (XAL) 19331 & - & - & - & - & - & $x$ & - & - & - & $x$ \\
\hline Alternanthera flava (L.) Mears (XAL) 16968 & - & - & - & - & - & $\mathrm{x}$ & - & - & $\mathrm{x}$ & - \\
\hline Amaranthus hybridus L. (XAL) 16472 & - & - & - & - & - & $\mathrm{x}$ & - & - & - & $\mathrm{x}$ \\
\hline Amaranthus spinosus L. (XAL) & - & - & - & - & - & $\mathrm{x}$ & - & - & - & $x$ \\
\hline Gomphrena filaginoides M.Martens et Galeotti (XAL) & - & - & - & - & - & $\mathrm{x}$ & - & - & - & $x$ \\
\hline Gomphrena nana (Stuchlik) Standl. (XAL) 19336 & - & - & - & - & - & $x$ & - & - & $\mathrm{x}$ & - \\
\hline Gomphrena serrata L. (XAL) 19476 & - & - & - & - & - & $x$ & _ & - & - & $\mathrm{x}$ \\
\hline Iresine calea (Ibáñez) Standl. (XAL) 19363 & - & - & - & - & $\mathrm{x}$ & - & - & - & $x$ & - \\
\hline Iresine celosia L. fo. ciliolata Suess. (XAL) 18265 & - & - & - & - & - & $\mathrm{x}$ & - & - & $x$ & - \\
\hline Iresine paniculata (L.) Kuntze (XAL) 16639 & - & - & - & - & - & $x$ & - & - & $x$ & - \\
\hline Iresine sp. 18148 (XAL) & - & - & - & - & - & $\mathrm{x}$ & - & - & - & $x$ \\
\hline Pfaffia hookeriana (Hemsl.) Greenm. (XAL) & - & - & - & - & - & $x$ & - & - & - & $x$ \\
\hline \multicolumn{11}{|l|}{ ANACARDIACEAE } \\
\hline Astronium graveolens Jacq. (XAL) 19451 & - & - & - & $\mathrm{x}$ & - & - & - & - & $x$ & - \\
\hline Comocladia engleriana Loes. (XAL) 18291 & - & - & - & $\mathrm{x}$ & - & - & - & - & $\mathrm{x}$ & - \\
\hline Mangifera indica L. (XAL) & $\mathrm{x}$ & - & - & $\mathrm{x}$ & - & - & - & - & - & $x$ \\
\hline Pistacia mexicana Kunth (XAL) 17138 & - & - & - & $\mathrm{x}$ & - & - & - & - & $x$ & - \\
\hline Rhus radicans $\mathrm{L}$. & - & - & - & - & $\mathrm{x}$ & - & _ & - & - & $\mathrm{x}$ \\
\hline Rhus terebinthifolia Schltdl. et Cham. (XAL) 17547 & - & - & - & - & $\mathrm{x}$ & - & - & - & $\mathrm{x}$ & - \\
\hline Spondias mombin L. (XAL) 19304 & - & - & - & $\mathrm{x}$ & - & - & - & - & - & $\mathrm{x}$ \\
\hline Spondias purpurea L. (XAL) & - & - & - & $\hat{x}$ & - & - & - & - & - & $x$ \\
\hline Toxicodendron radicans (L.) Kuntze (XAL) 18479 & - & - & - & - & $x$ & - & - & - & - & $x$ \\
\hline \multicolumn{11}{|l|}{ ANNONACEAE } \\
\hline Annona globiflora Schltdl. (XAL) 18286 & - & - & - & $\mathrm{x}$ & - & - & - & - & $x$ & - \\
\hline \multicolumn{11}{|l|}{ APOCYNACEAE } \\
\hline Echites mexicanus (Müll.Arg.) Miers (XAL) & - & - & - & - & - & - & $\mathrm{x}$ & - & - & $\mathrm{x}$ \\
\hline Fernaldia pandurata (A.DC.) Woodson (XAL) 19272 & - & - & - & - & - & - & $\mathrm{x}$ & - & - & $\mathrm{x}$ \\
\hline Mandevilla donnell-smithii Woodson (XAL) & - & - & - & - & - & - & $\hat{x}$ & - & $x$ & - \\
\hline Mandevilla karwinskii (Müll.Arg.) Hemsl. (XAL) & - & - & - & - & - & - & $\mathrm{x}$ & - & - & $x$ \\
\hline Plumeria rubra L. (XAL) $17576^{\circ}$ & - & - & - & $\mathrm{x}$ & - & - & - & - & $\mathrm{x}$ & - \\
\hline Prestonia mexicana A.DC. (XAL) 18033 & - & - & - & - & - & - & $\mathrm{x}$ & - & - & $x$ \\
\hline Rauvolfia tetraphylla L. (XAL) & - & - & - & - & $\mathrm{x}$ & - & - & - & - & $x$ \\
\hline Stemmadenia obovata K.Schum. (XAL) 16315 & - & - & - & $\mathrm{x}$ & - & - & - & - & - & $\mathrm{x}$ \\
\hline Tabernaemontana alba Mill. (XAL) 16556 & - & - & - & $\mathrm{x}$ & - & - & - & - & - & $x$ \\
\hline Tabernaemontana sp. (XAL) 16646 & - & - & - & $x$ & - & - & - & - & $\mathrm{x}$ & - \\
\hline Thevetia peruviana (Pers.) K.Schum. (XAL) & - & - & - & $x$ & - & - & - & - & - & $x$ \\
\hline Tonduzia longifolia (A.DC.) Woodson (XAL) 16645 & - & - & - & $\mathrm{x}$ & - & - & - & - & $\mathrm{x}$ & - \\
\hline Urechites andrieuxii Müll.Arg. (XAL) 17539 & - & - & - & - & - & - & $\mathrm{x}$ & - & $\mathrm{x}$ & - \\
\hline \multicolumn{11}{|l|}{ ARALIACEAE } \\
\hline Dendropanax arboreus (L.) Decne. et Planch. (XAL) & - & - & - & $\mathrm{x}$ & - & - & - & - & - & $\mathrm{x}$ \\
\hline \multicolumn{11}{|l|}{ ASCLEPIADACEAE } \\
\hline \multirow{2}{*}{\multicolumn{11}{|c|}{ Cynanchum schlechtendalii (Decne.) Standl. et }} \\
\hline & & & & & & & & & & \\
\hline Steyerm. (XAL) 17246 & - & - & - & - & - & - & $\mathrm{x}$ & - & $\mathrm{x}$ & - \\
\hline Gonolobus barbatus Kunth (XAL) & - & - & - & - & - & - & $x$ & - & - & $x$ \\
\hline Gonolobus chloranthus Schltdl. (XAL) 18019 & - & - & - & - & - & - & $\mathrm{x}$ & - & $\mathrm{x}$ & - \\
\hline Marsdenia coulteri Hemsl. (XAL) 19220 & - & - & - & - & - & - & $x$ & - & $x$ & - \\
\hline Matelea prosthecidiscus Woodson (XAL) 18000 & - & - & - & - & - & - & $\mathrm{x}$ & - & $\mathrm{x}$ & - \\
\hline Matelea velutina (Schltdl.) Woodson (XAL) 18233 & - & - & - & - & - & - & $\mathrm{x}$ & - & - & $\mathrm{x}$ \\
\hline Metastelma schlechtendalii Decne. (XAL) 19055 & - & - & - & - & - & - & $\hat{x}$ & - & $x$ & - \\
\hline \multicolumn{11}{|l|}{ ASTERACEAE } \\
\hline Acmella radicans (Jacq.) R.K.Jansen (XAL) 18801 & - & - & - & - & - & $\mathrm{x}$ & - & - & - & $x$ \\
\hline
\end{tabular}


Adenophyllum porophyllum (Cav.) Hemsl. (XAL) 16367

Ageratina chiapensis (B.L.Rob.) R.M.King et H.Rob. (XAL) 17312

Ageratina havanensis (Kunth) R.M.King et H.Rob. (XAL) 17044

Ageratina ligustrina (DC.) R.M.King et H.Rob. (XAL) 19158

Aldama dentata La Llave (XAL)

Baltimora geminata (Brandegee) Stuessy (XAL) 18419

Baltimora recta L. (XAL) 18162

Bidens pilosa L. (XAL) 19317

Bidens reptans (L.) G.Don (XAL) 18123

Brickellia sp. (XAL) 18610

Calea ternifolia Kunth var. ternifolia (XAL)

Calea urticifolia (Mill.) DC. (XAL) 16409

Calyptocarpus vialis Less. (XAL)

Chaptalia nutans (L.) Pol. (XAL) 19001

Chromolaena collina (DC.) R.M.King et H.Rob.

(XAL)

Cirsium mexicanum DC. (XAL) 16294

Conyza canadensis (L.) Cronquist (XAL) 19357

Delilia berteroi Spreng. (XAL) 18397

Eclipta alba (L.) Hassk. (XAL)

Eupatorium arsenei B.L Rob. (XAL) 16697

Eupatorium odoratum L. (XAL) 16649

Fleischmannia pycnocephala (Less.) R.M.King et H.Rob. (XAL)

Galeana pratensis (Kunth) Rydb. (XAL) 18540

Galinsoga quadriradiata Ruiz et Pav. (XAL) 16681

Koanophyllon galeottii (B.L.Rob.) R.M.King et H.Rob. (XAL) 16641

Lagascea mollis Cav. (XAL) 18229

Loxothysanus sinuatus (Less.) B.L.Rob. (XAL) 17046

Melampodium divaricatum (Rich.) DC. (XAL) 19497

Melampodium gracile Less. (XAL)

Melampodium microcephalum Less. (XAL)

Montanoa tomentosa Cerv. subsp. xanthiifolia

(Sch.Bip. ex C.Koch) V.A.Funk (XAL) 19425

Parthenium hysterophorus L. (XAL) 19316

Pectis prostrata Cav. (XAL) 19298

Pluchea sp. (XAL)

Polymnia maculata Cav. (XAL)

Porophyllum macrocephalum (DC.) (XAL) 19328

Porophyllum punctatum (Mill.) S.F.Blake (XAL) 19343

Pseudelephantopus spicatus (B.Juss. ex Aubl.)

C.F.Baker (XAL) 19354

Roldana sartorii (Sch.Bip. ex Hemsl.) H.Rob. et Brettell (XAL)

Sanvitalia procumbens Lam. (XAL) 18024

Sclerocarpus divaricatus (Benth.) Benth. et Hook.f. ex Hemsl. (XAL) 19292

Sclerocarpus uniserialis (Hook.) Benth. et Hook.f. ex Hemsl. (XAL) 


\begin{tabular}{|c|c|c|c|c|c|c|c|c|c|c|}
\hline Familia y especie & $\mathrm{C}$ & EM & EV & A & $\operatorname{Ar}$ & $\mathrm{H}$ & B & $\mathrm{E}$ & $\mathrm{P}$ & $\mathrm{S}$ \\
\hline Schkuhria pinnata (Lam.) Kuntze ex Thell. (XAL) & - & - & - & - & - & $x$ & - & - & - & $x$ \\
\hline Senecio cinerarioides Kunth (XAL) 16280 & - & - & - & - & $x$ & - & - & - & $x$ & - \\
\hline Senecio deppeanus Hemsl. (XAL) 18675 & - & - & - & - & - & $\mathrm{x}$ & - & - & $\mathrm{x}$ & - \\
\hline Senecio sartorii Sch.Bip. ex Hemsl. (XAL) 17316 & - & - & - & - & $x$ & - & - & - & $\mathrm{x}$ & - \\
\hline Senecio sp. (XAL) 16947 & - & - & - & - & $\mathrm{x}$ & - & - & - & $\mathrm{x}$ & - \\
\hline Simsia amplexicaulis (Cav.) Pers. (XAL) 16477 & - & - & - & - & - & $x$ & - & - & - & $x$ \\
\hline Simsia calva A. Gray (XAL) 19338 & - & - & - & - & - & $\mathrm{x}$ & - & - & $\mathrm{x}$ & - \\
\hline Sonchus oleraceus L. (XAL) 18978 & - & - & - & - & - & $\mathrm{x}$ & - & - & - & $x$ \\
\hline Tithonia tubiformis (Jacq.) Cass. (XAL) 18436 & - & - & - & - & - & $\mathrm{x}$ & - & - & - & $x$ \\
\hline Tridax procumbens L. (XAL) 16638 & - & - & - & - & - & $\mathrm{x}$ & _ & - & - & $\mathrm{x}$ \\
\hline \multicolumn{11}{|l|}{ Trigonospermum annuum McVaugh et Lask. (XAL) } \\
\hline 19294 & - & - & - & - & - & $\mathrm{x}$ & - & - & $\mathrm{x}$ & - \\
\hline Trixis inula Crantz (XAL) 17989 & - & - & - & - & $x$ & - & - & - & $x$ & - \\
\hline Verbesina crocata (Cav.) Less. (XAL) 19426 & - & - & - & - & $\mathrm{x}$ & - & - & - & $\mathrm{x}$ & - \\
\hline Verbesina gigantea Jacq. (XAL) 16515 & - & - & - & - & $\mathrm{x}$ & - & - & - & $\mathrm{x}$ & - \\
\hline Verbesina gigantoides B.L.Rob. (XAL) 17241 & - & - & - & - & $\mathrm{x}$ & - & - & - & $\mathrm{x}$ & - \\
\hline Verbesina lindenii (Sch.Bip.) S.F.Blake (XAL) 19379 & - & - & - & - & $\mathrm{x}$ & - & - & - & $\mathrm{x}$ & - \\
\hline Verbesina ovatifolia A.Gray (XAL) 16308 & - & - & - & - & $\mathrm{x}$ & - & - & - & $\mathrm{x}$ & - \\
\hline Vernonia sp. (XAL) 17550 & - & - & - & - & $\mathrm{x}$ & - & - & - & $\mathrm{x}$ & - \\
\hline Vernonia triflosculosa Kunth (XAL) 16970 & - & - & - & $\mathrm{x}$ & - & - & - & - & $\mathrm{x}$ & - \\
\hline Viguiera cordata (Hook. et Arn.) D'Arcy (XAL) & - & - & - & - & - & $\mathrm{x}$ & - & - & $\mathrm{x}$ & - \\
\hline \multicolumn{11}{|l|}{ Zinnia americana (Mill.) Olorode et A.M.Torres } \\
\hline$(\mathrm{XAL})$ & - & - & - & - & - & $x$ & - & - & - & $x$ \\
\hline Zinnia peruviana (L.) L. (XAL) 19475 & - & - & - & - & - & $\mathrm{x}$ & - & - & - & $x$ \\
\hline \multicolumn{11}{|l|}{ BEGONIACEAE } \\
\hline Begonia heracleifolia Schltdl. et Cham. (XAL) 17219 & - & - & - & - & - & $x$ & - & - & $x$ & - \\
\hline Begonia hydrocotylifolia Otto ex Hook. (XAL) & - & - & $\mathrm{x}$ & - & - & $\mathrm{x}$ & - & - & $\mathrm{x}$ & - \\
\hline Begonia peltata Otto et D.Dietr. (XAL) 18014 & - & - & - & - & - & $x$ & - & - & $x$ & - \\
\hline \multicolumn{11}{|l|}{ BETULACEAE } \\
\hline \multicolumn{11}{|l|}{ Alnus acuminata Kunth subsp. arguta (Schltdl.) Furlow } \\
\hline \multicolumn{11}{|l|}{ BIGNONIACEAE } \\
\hline Amphilophium paniculatum (L.) Kunth (XAL) 19342 & - & - & - & - & - & - & $\mathrm{x}$ & - & $\mathrm{x}$ & - \\
\hline Cydista heterophylla Seibert (XAL) & - & - & - & - & - & - & $\mathrm{x}$ & - & - & $\mathrm{x}$ \\
\hline Melloa quadrivalvis (Jacq.) A.H.Gentry (XAL) & - & - & - & - & - & - & $\mathrm{x}$ & - & $\mathrm{x}$ & - \\
\hline Stizophyllum riparium (Kunth) Sandwith (XAL) & - & - & - & - & - & - & $\mathrm{x}$ & - & - & $\mathrm{x}$ \\
\hline Tecoma stans (L.) Juss. ex Kunth (XAL) 19015 & - & - & - & $\mathrm{x}$ & - & - & - & - & - & $\mathrm{x}$ \\
\hline \multicolumn{11}{|l|}{ BOMBACACEAE } \\
\hline \multicolumn{11}{|l|}{ Ceiba aesculifolia (Kunth) Britten et Baker f. (XAL) } \\
\hline 17574 & - & - & - & $\mathrm{x}$ & - & - & - & - & $\mathrm{x}$ & - \\
\hline Pseudobombax ellipticum (Kunth) Dugand var. & & & & & & & & & & \\
\hline tenuiflorum A.Robyns (XAL) $16663^{\circ}$ & - & $\mathrm{x}$ & - & $\mathrm{x}$ & - & - & - & - & $\mathrm{x}$ & - \\
\hline \multicolumn{11}{|l|}{ BORAGINACEAE } \\
\hline Cordia alliodora (Ruiz et Pav.) Oken (XAL) 19017 & - & - & - & $\mathrm{x}$ & - & - & - & - & - & $\mathrm{x}$ \\
\hline Cordia ambigua Schltdl. et Cham. (XAL) 18275 & - & - & - & - & $x$ & - & - & - & $\mathrm{x}$ & - \\
\hline Cordia foliosa M.Martens et Galeotti (XAL) & - & - & - & - & $x$ & - & - & - & - & $x$ \\
\hline Cordia podocephala Torr. (XAL) & - & - & - & - & $x$ & - & _ & - & - & $\mathrm{x}$ \\
\hline Cordia pringlei B.L.Rob. (XAL) 18377 & - & - & - & - & $x$ & - & - & - & - & $x$ \\
\hline Cordia sp. (XAL) & - & - & - & - & $x$ & - & _ & - & - & $\mathrm{x}$ \\
\hline Cordia spinescens $\mathrm{L} .(\mathrm{XAL})$ & - & - & - & - & $x$ & - & - & - & - & $x$ \\
\hline Ehretia tinifolia L. (XAL) 16550 & - & - & - & $\mathrm{x}$ & - & - & - & - & - & $\mathrm{x}$ \\
\hline Heliotropium procumbens Mill. (XAL) & - & - & - & - & - & $x$ & - & - & - & $x$ \\
\hline Heliotropium sp. (XAL) 19249 & - & - & - & - & - & $\mathrm{x}$ & - & - & - & $x$ \\
\hline Tournefortia densiflora M.Martens et Galeotti & - & - & - & - & - & - & $\mathrm{x}$ & - & - & $x$ \\
\hline Tournefortia volubilis L. (XAL) 18368 & - & - & - & - & - & - & $\mathrm{x}$ & - & $\mathrm{x}$ & - \\
\hline
\end{tabular}




\begin{tabular}{|c|c|c|c|c|c|c|c|c|c|c|}
\hline Familia y especie & C & EM & EV & A & $\operatorname{Ar}$ & $\mathrm{H}$ & B & $\mathrm{E}$ & $P$ & $\mathrm{~S}$ \\
\hline \multicolumn{11}{|l|}{ BRASSICACEAE } \\
\hline Lepidium virginicum L. (XAL) 18842 & - & - & - & - & - & $\mathrm{x}$ & - & - & - & $\mathrm{x}$ \\
\hline \multicolumn{11}{|l|}{ BURSERACEAE } \\
\hline \multirow{2}{*}{\multicolumn{11}{|c|}{ Bursera fagaroides (Kunth) Engl. var. fagaroides }} \\
\hline & & & & & & & & & & \\
\hline $\begin{array}{l}\text { (XAL) } 16297 \\
\text { Bursera simaruba (L.) Sarg. (XAL) } 16318\end{array}$ & - & $\mathrm{X}$ & - & - & $\mathrm{X}$ & - & - & - & $\begin{array}{l}X \\
y\end{array}$ & - \\
\hline \multicolumn{11}{|l|}{ CACTACEAE } \\
\hline Acanthocereus sp. 19393 (XAL) & - & - & - & - & - & $x$ & - & - & $x$ & - \\
\hline \multicolumn{11}{|l|}{ Cephalocereus palmeri Rose var. palmeri (XAL) } \\
\hline 19395 & - & $x$ & - & - & $x$ & - & - & - & $\mathrm{x}$ & - \\
\hline \multicolumn{11}{|l|}{ Cephalocereus palmeri Rose var. sartorianus (Rose) } \\
\hline Krainz (XAL) 18344 & - & $x$ & - & - & $x$ & - & - & - & $\mathrm{x}$ & - \\
\hline Epiphyllum oxypetalum (DC.) Haw. (XAL) 17984 & - & - & - & - & - & - & - & $\mathrm{x}$ & $\mathrm{X}$ & - \\
\hline Hylocereus sp. (XAL) 19396b & - & - & - & - & - & $\mathrm{x}$ & - & - & $\mathrm{X}$ & - \\
\hline Mammillaria eriacantha Link et Otto (XAL) 17561 & - & - & $\mathrm{X}$ & - & - & $\mathrm{x}$ & - & - & $\mathrm{x}$ & - \\
\hline \multicolumn{11}{|l|}{ Neobuxbaumia scoparia (Poselger) Backeb. (XAL) } \\
\hline 18010 & - & $x$ & - & $\mathrm{x}$ & - & - & - & - & $\mathrm{x}$ & - \\
\hline \multicolumn{11}{|l|}{ Nopalea dejecta (Salm-Dyck) Salm-Dyck (XAL) } \\
\hline 17192 & - & - & - & - & $x$ & - & - & - & $\mathrm{x}$ & - \\
\hline Opuntia sp. 17110 (XAL) & - & - & - & - & - & $\mathrm{x}$ & - & - & $x$ & - \\
\hline Rhipsalis baccifera (J.S.Muell.) Stearn (XAL) 17987 & - & - & - & - & - & - & - & $\mathrm{x}$ & $\mathrm{x}$ & - \\
\hline $\begin{array}{l}\text { Selenicereus coniflorus (Weing.) Britton } \\
\text { et Rose (XAL) } 17032\end{array}$ & - & $x$ & - & - & - & $x$ & - & - & $x$ & - \\
\hline \multicolumn{11}{|l|}{ Selenicereus grandiflorus (L.) Britton et Rose (XAL) } \\
\hline 19225 & - & - & - & - & - & $x$ & - & - & $x$ & - \\
\hline \multicolumn{11}{|l|}{ Selenicereus testudo (Karw. ex Zucc.) Buxb. (XAL) } \\
\hline 17891 & - & - & - & - & - & $\mathrm{x}$ & - & - & $\mathrm{x}$ & - \\
\hline \multirow{2}{*}{\multicolumn{11}{|c|}{ CAMPANULACEAE }} \\
\hline & & & & & & & & & & \\
\hline Lobelia xalapensis Kunth (XAL) & - & - & - & - & - & $\mathrm{x}$ & - & - & - & $\mathrm{x}$ \\
\hline \multicolumn{11}{|l|}{ CAPPARIDACEAE } \\
\hline Capparis sp. (XAL) 16549 & - & - & - & $\mathrm{x}$ & - & - & - & - & $\mathrm{X}$ & - \\
\hline Capparis pringlei Briq. (XAL) 19466 & - & - & - & $x$ & - & - & - & - & $x$ & - \\
\hline \multicolumn{11}{|l|}{ CARYOPHYLLACEAE } \\
\hline Drymaria cordata (L.) Willd. ex Schult. (XAL) 17508 & - & - & - & - & - & $\mathrm{x}$ & - & - & - & $\mathrm{x}$ \\
\hline Drymaria villosa Schtldl. et Cham. subsp. palustris & - & - & - & - & - & $\mathrm{x}$ & - & - & - & $\mathrm{x}$ \\
\hline \multicolumn{11}{|l|}{ Drymaria villosa Schltdl. et Cham. subsp. villosa } \\
\hline$(\mathrm{XAL})$ & - & - & - & - & - & $\mathrm{x}$ & - & - & - & $\mathrm{x}$ \\
\hline \multicolumn{11}{|l|}{ CELASTRACEAE } \\
\hline Microtropis schiedeana Loes. (XAL) 17167 & - & - & - & $x$ & - & - & - & - & - & $\mathrm{x}$ \\
\hline Myginda uragoga Jacq. (XAL) 16566 & - & - & - & - & $\mathrm{x}$ & - & - & - & - & $\mathrm{x}$ \\
\hline Schaefferia frutescens Jacq. (XAL) 19016 & - & - & - & - & $\mathrm{x}$ & - & - & - & - & $\mathrm{x}$ \\
\hline Wimmeria concolor Schltdl. et Cham. (XAL) 17527 & - & - & - & - & $\mathrm{x}$ & - & - & - & $\mathrm{X}$ & - \\
\hline Wimmeria pubescens Radlk. (XAL) 19521 & - & - & - & $\mathrm{x}$ & - & - & - & - & - & $\mathrm{x}$ \\
\hline \multicolumn{11}{|l|}{ CHENOPODIACEAE } \\
\hline Chenopodium ambrosioides L. (XAL) 16870 & - & - & - & - & - & $x$ & - & - & - & $x$ \\
\hline CLUSIACEAE & & & & & & & & & & \\
\hline Clusia lundellii Standl. (XAL) 17286 & - & - & - & $\mathrm{x}$ & - & - & - & - & $\mathrm{x}$ & - \\
\hline Clusia mexicana Vesque (XAL) 17557 & - & - & - & $x$ & - & - & - & - & $\mathrm{x}$ & - \\
\hline Clusia quadrangula Bartlett (XAL) 17075 & - & - & - & $\mathrm{x}$ & - & - & - & - & $\mathrm{x}$ & - \\
\hline COCHLOSPERMACEAE & & & & & & & & & & \\
\hline Cochlospermum vitifolium (Willd.) Spreng. (XAL) & & & & & & & & & & \\
\hline 18105 & - & - & - & $x$ & - & - & - & - & $x$ & - \\
\hline
\end{tabular}




\section{COMBRETACEAE}

Combretum fruticosum (Loefl.) Stuntz (XAL) 19423 CONVOLVULACEAE

Dichondra sericea Sw. (XAL) 16739

Evolvulus alsinoides (L.) L. (XAL) 16618

Ipomoea alba L. (XAL)

Ipomoea batatas (L.) Lam. (XAL) 18245

Ipomoea indica (Burm.) Merr. (XAL)

Ipomoea jalapa (L.) Pursh (XAL) 18168

Ipomoea nil (L.) Roth (XAL) 19339

Ipomoea sp. (XAL)

Ipomoea wolcottiana Rose var. wolcottiana (XAL) 17566

Merremia dissecta (Jacq.) Hallier f. (XAL)

Merremia quinquefolia (L.) Hallier f. (XAL) 19325

\section{CRASSULACEAE}

Echeveria lurida Haw. (XAL) 16629

Echeveria racemosa Schltdl. et Cham.

Kalanchoe pinnata (Lam.) Pers. (XAL) 18489

Sedum adolphii Hamet (XAL) 19214

Sedum nussbaumerianum Bitter (XAL) 17051

\section{CUCURBITACEAE}

Cucumis anguria L. (XAL)

Cucumis melo L. (XAL) 19344

Doyerea emetocathartica Grosourdy (XAL) 19228

Luffa cylindrica M.Roem. (XAL)

Melothria pendula L.

\section{CUNONIACEAE}

Weinmannia intermedia Schltdl. et Cham. (XAL)

\section{3}

\section{EBENACEAE}

Diospyros oaxacana Standl. (XAL) 16674

Diospyros verae-crucis (Standl.) Standl. (XAL) 16323

\section{ERYTHROXYLACEAE}

Erythroxylum havanense Jacq. (XAL) 16307

\section{EUPHORBIACEAE}

Acalypha adenostachya Müll.Arg. (XAL) 16688

Acalypha alopecuroides Jacq. (XAL) 19326

Acalypha arvensis Poepp. et Endl. (XAL)

Acalypha deppeana Schltr. (XAL) 18350

Acalypha diversifolia Jacq. (XAL) 18283

Acalypha schiedeana Schltdl. (XAL)

Acalypha setosa A.Rich. (XAL)

Acalypha unibracteata Müll.Arg. (XAL) 19233

Adelia barbinervis Cham. et Schltdl. (XAL) 19404

Argythamnia guatemalensis Müll.Arg. (XAL)

Argythamnia sp. (XAL)

Bernardia interrupta (Schltdl.) Müll.Arg. (XAL) 19375

Bernardia mexicana (Hook. et Arn.) Müll.Arg. (XAL) 16679

Bernardia sp.

Chamaesyce ammannioides (Kunth) Small (XAL) 16378

Chamaesyce dioica (Kunth) Millsp. (XAL) 16757

Chamaesyce hirta (L.) Millsp. (XAL) 18030 


\begin{tabular}{|c|c|c|c|c|c|c|c|c|c|c|}
\hline Familia y especie & $\mathrm{C}$ & EM & EV & A & $\operatorname{Ar}$ & $\mathrm{H}$ & B & $\mathrm{E}$ & $\mathrm{P}$ & $\mathrm{S}$ \\
\hline Chamaesyce hypericifolia (L.) Millsp. (XAL) 19299 & - & - & - & - & - & $x$ & - & - & - & $x$ \\
\hline \multicolumn{11}{|l|}{ Chamaesyce lasiocarpa (Klotzsch) Arthur (XAL) } \\
\hline 18166 & - & - & - & - & - & $\mathrm{x}$ & - & - & - & $\mathrm{x}$ \\
\hline Chamaesyce prostrata (Aiton) Small (XAL) 18920 & - & - & - & - & - & $\mathrm{x}$ & - & - & - & $\mathrm{x}$ \\
\hline \multicolumn{11}{|l|}{ Cnidoscolus aconitifolius (Mill.) I.M.Johnst. (XAL) } \\
\hline 16644 & - & - & - & - & $x$ & - & - & - & $\mathrm{x}$ & - \\
\hline Croton ciliatoglandulosus Ortega (XAL) 16643 & - & - & - & - & $\mathrm{X}$ & - & - & - & $\mathrm{x}$ & - \\
\hline Croton cortesianus Kunth (XAL) 16476 & - & - & - & - & $x$ & - & - & - & - & $\mathrm{x}$ \\
\hline Croton glandulosus L. (XAL) 16643 & - & - & - & - & - & $\mathrm{x}$ & - & - & - & $\mathrm{x}$ \\
\hline Croton hirtus L’Hér. (XAL) 18519 & - & - & - & - & - & $\mathrm{x}$ & - & - & - & $\mathrm{x}$ \\
\hline Croton miradorensis Müll.Arg. (XAL) 18175 & - & - & - & $x$ & - & - & - & - & - & $\mathrm{x}$ \\
\hline Croton niveus Jacq. (XAL) 19065 & - & - & - & - & $\mathrm{x}$ & - & - & - & - & $\mathrm{x}$ \\
\hline Croton sp. (XAL) 18237 & - & - & - & - & $x$ & - & - & - & - & $x$ \\
\hline Dalechampia cissifolia Poepp. et Endl. (XAL) 19407 & - & - & - & - & - & - & $\mathrm{X}$ & - & $\mathrm{X}$ & - \\
\hline Dalechampia sp. (XAL) 16632 & - & - & - & - & - & - & $x$ & - & $\mathrm{x}$ & - \\
\hline Dalechampia scandens L. (XAL) 16632 & - & - & - & - & - & - & $\mathrm{x}$ & - & $\mathrm{x}$ & - \\
\hline Euphorbia cyathophora Murr (XAL) 19386 & - & - & - & - & - & $\mathrm{x}$ & - & - & $\mathrm{x}$ & - \\
\hline Euphorbia dentata Michx. (XAL) 16640 & - & - & - & - & - & $\mathrm{x}$ & - & - & $\mathrm{x}$ & - \\
\hline Euphorbia graminea Jacq. (XAL) 19516 & - & - & - & - & - & $\mathrm{x}$ & - & - & - & $\mathrm{x}$ \\
\hline Euphorbia heterophylla L. (XAL) 16698 & - & - & - & - & - & $\mathrm{x}$ & - & - & - & $\mathrm{x}$ \\
\hline Euphorbia ixtlana Huft (XAL) & - & - & - & - & - & $\mathrm{x}$ & - & - & $\mathrm{X}$ & - \\
\hline Euphorbia schlechtendalii Boiss. (XAL) 16615 & - & - & - & - & $\mathrm{x}$ & - & - & - & $\mathrm{x}$ & - \\
\hline Pedilanthus sp. (XAL) 18426 & - & - & - & - & - & $\mathrm{x}$ & - & - & $x$ & - \\
\hline \multicolumn{11}{|l|}{ Phyllanthus amarus Schumach. et Thonn. (XAL) } \\
\hline 18035 & - & - & - & - & - & $\mathrm{x}$ & - & - & - & $\mathrm{x}$ \\
\hline Phyllanthus caroliniensis Walter (XAL) 19387 & - & - & - & - & - & $\mathrm{x}$ & - & - & $\mathrm{x}$ & - \\
\hline Phyllanthus ferax Standl. (XAL) 19090 & - & - & - & - & - & $\mathrm{x}$ & - & - & - & $\mathrm{x}$ \\
\hline Phyllanthus niruri L. (XAL) 19332 & - & - & - & - & - & $\mathrm{x}$ & - & - & - & $\mathrm{x}$ \\
\hline Ricinus communis L. (XAL) & - & - & - & - & $\mathrm{x}$ & - & - & - & - & $\mathrm{x}$ \\
\hline Tragia brevispica Engelm. et A.Gray (XAL) & - & - & - & - & - & - & $\mathrm{x}$ & - & - & $\mathrm{x}$ \\
\hline Tragia glanduligera Pax et K.Hoffm. (XAL) 17206 & - & - & - & - & - & $x$ & - & - & - & $x$ \\
\hline \multicolumn{11}{|l|}{ FABACEAE } \\
\hline Acacia cornigera (L.) Willd. (XAL) 16553 & - & - & - & - & $\mathrm{X}$ & - & - & - & - & $\mathrm{x}$ \\
\hline Acacia farnesiana (L.) Willd. (XAL) 16555 & - & - & - & - & $\mathrm{x}$ & - & - & - & - & $\mathrm{x}$ \\
\hline Acacia hayesii Benth. (XAL) 19440 & - & - & - & - & $x$ & - & - & - & $\mathrm{x}$ & - \\
\hline \multicolumn{11}{|l|}{ Acacia pennatula (Schltdl. et Cham.) Benth. (XAL) } \\
\hline 16696 & - & - & - & $\mathrm{x}$ & - & - & - & - & - & $\mathrm{x}$ \\
\hline \multicolumn{11}{|l|}{ Aeschynomene americana L. var. flabellata Rudd } \\
\hline$(\mathrm{XAL})$ & - & - & - & - & - & $\mathrm{x}$ & - & - & - & $\mathrm{x}$ \\
\hline Aeschynomene elegans Cham. et Schltdl. (XAL) & - & - & - & - & - & $\mathrm{x}$ & - & - & - & $\mathrm{x}$ \\
\hline \multicolumn{11}{|l|}{ Aeschynomene fascicularis Schltdl. et Cham. (XAL) } \\
\hline 19486 & - & - & - & - & - & $\mathrm{x}$ & - & - & - & $\mathrm{x}$ \\
\hline Bauhinia divaricata L. (XAL) 18159 & - & - & - & - & $\mathrm{x}$ & - & - & - & - & $\mathrm{x}$ \\
\hline Brongniartia aff. intermedia Moric. (XAL) 20146 & - & - & - & - & $x$ & - & - & - & - & $\mathrm{x}$ \\
\hline \multirow{2}{*}{\multicolumn{11}{|c|}{ Calliandra rubescens (M.Martens et Galeotti) Standl. }} \\
\hline & - & - & - & - & $x$ & - & - & - & - & $\mathrm{x}$ \\
\hline Canavalia villosa Benth. (XAL) & - & - & - & - & - & - & $\mathrm{x}$ & - & $\mathrm{x}$ & - \\
\hline Centrosema virginianum (L.) Benth. (XAL) 16634 & - & - & - & - & - & - & $\mathrm{x}$ & - & $\mathrm{X}$ & - \\
\hline \multicolumn{11}{|l|}{ Chamaecrista hispidula (Vahl) H.S.Irwin et Barneby } \\
\hline (XAL) 17337 & - & - & - & - & - & $\mathrm{x}$ & - & - & - & $\mathrm{x}$ \\
\hline Chamaecrista nictitans (L.) Moench (XAL) 19322 & - & - & - & - & - & $\mathrm{x}$ & - & - & - & $\mathrm{x}$ \\
\hline Cracca caribaea (Jacq.) Benth. (XAL) 18226 & - & - & - & - & $\mathrm{x}$ & - & - & - & $\mathrm{x}$ & - \\
\hline Crotalaria incana L. (XAL) 19350 & - & - & - & - & - & $\mathrm{x}$ & - & - & - & $\mathrm{x}$ \\
\hline \multicolumn{11}{|l|}{ Dalea carthagenensis (Jacq.) J.F.Macbr. var. barbata } \\
\hline (Oerst.) Barneby (XAL) & - & - & - & - & - & $\mathrm{x}$ & - & - & - & $\mathrm{x}$ \\
\hline
\end{tabular}


Gonzalo Castillo-Campos, Patricia Dávila-Aranda y José Alejandro Zavala-Hurtado

\begin{tabular}{|c|c|c|c|c|c|c|c|c|c|c|}
\hline Familia y especie & $\mathrm{C}$ & EM & EV & A & $\mathrm{Ar}$ & $\mathrm{H}$ & B & $\mathrm{E}$ & $\mathrm{P}$ & $\mathrm{s}$ \\
\hline Dalea cliffortiana Willd. (XAL) & - & - & - & - & - & $x$ & - & - & - & $x$ \\
\hline \multicolumn{11}{|l|}{ Dalea foliolosa (Aiton) Barneby var. citrina (Rydb.) } \\
\hline Barneby (XAL) 19477 & - & - & - & - & - & $\mathrm{x}$ & - & - & - & $\mathrm{x}$ \\
\hline \multicolumn{11}{|l|}{ Dalea scandens (Mill.) R.T.Clausen var. paucifolia } \\
\hline (J.M.Coult.) Barneby (XAL) & - & - & - & - & $\mathrm{x}$ & - & - & - & - & $\mathrm{x}$ \\
\hline Dalea sp. (XAL) & - & - & - & - & - & $\mathrm{x}$ & - & - & - & $\mathrm{x}$ \\
\hline Dalea tomentosa (Cav.) Willd. (XAL) & - & - & - & - & - & $\mathrm{x}$ & - & - & - & $x$ \\
\hline Desmanthus pubescens B.L.Turner (XAL) 16690 A & - & - & - & - & - & $\mathrm{x}$ & - & - & - & $x$ \\
\hline \multirow{2}{*}{\multicolumn{11}{|c|}{ Desmanthus virgatus (L.) Willd. var. depressus (Humb. }} \\
\hline et Bonpl. ex Willd.) B.L.Turner (XAL) 19285 & & - & ـ & - & - & $\mathrm{x}$ & - & _ & - & $\mathrm{x}$ \\
\hline Desmodium adscendens (Sw.) DC. (XAL) 16984 & - & - & - & - & - & $\mathrm{x}$ & - & - & - & $\mathrm{x}$ \\
\hline Desmodium distortum (Aubl.) J.F.Macbr. (XAL) 18285 & - & - & - & - & - & $\mathrm{x}$ & - & - & $\mathrm{x}$ & - \\
\hline Desmodium helleri Peyr. (XAL) 17532 & - & - & - & - & - & $\mathrm{x}$ & - & - & - & $\mathrm{x}$ \\
\hline Desmodium incanum DC. (XAL) 19103 & - & - & - & - & - & $\mathrm{x}$ & - & - & - & $\mathrm{x}$ \\
\hline Desmodium neomexicanum A.Gray (XAL) 19431 & - & - & - & - & - & $\mathrm{x}$ & - & - & $\mathrm{x}$ & - \\
\hline Desmodium prehensile Schltdl. (XAL) & - & - & - & - & - & $\mathrm{x}$ & - & - & - & $\mathrm{x}$ \\
\hline Desmodium procumbens (Mill.) Hitchc. (XAL) 18163 & - & - & - & - & - & $\mathrm{x}$ & - & - & $\mathrm{x}$ & - \\
\hline \multicolumn{11}{|l|}{ Desmodium procumbens (Mill.) Hitchc. var. longipes } \\
\hline (Schindl) B.G.Schub. (XAL) & - & - & - & - & - & $x$ & - & - & $x$ & - \\
\hline \multicolumn{10}{|l|}{ Desmodium procumbens (Mill.) Hitchc. var. } & \\
\hline (XAL) & - & - & - & - & - & $\mathrm{x}$ & - & - & $\mathrm{x}$ & - \\
\hline Desmodium scorpiurus (Sw.) Desv. (XAL) 19340 & - & - & - & - & - & $\mathrm{x}$ & - & - & - & $\mathrm{x}$ \\
\hline Desmodium sessilifolium Torr. et A.Gray (XAL) & - & - & - & - & - & $\mathrm{x}$ & - & - & - & $x$ \\
\hline Desmodium tortuosum (Sw.) DC. (XAL) 19301 & - & - & - & - & - & $\mathrm{x}$ & - & - & - & $x$ \\
\hline Diphysa americana (Mill.) M.Sousa (XAL) & - & - & - & $\mathrm{x}$ & - & - & - & - & - & $\mathrm{x}$ \\
\hline Diphysa robinioides Benth. (XAL) 16576 & - & - & - & - & $x$ & - & - & - & $\mathrm{x}$ & - \\
\hline \multicolumn{11}{|l|}{ Enterolobium cyclocarpum (Jacq.) Griseb. (XAL) } \\
\hline 16413 & - & - & - & $\mathrm{x}$ & - & - & - & - & - & $\mathrm{x}$ \\
\hline Erythrina americana Mill. (XAL) 17523 & - & - & - & $\mathrm{x}$ & - & - & - & - & $\mathrm{x}$ & - \\
\hline Eysenhardtia polystachya (Ortega) Sarg. (XAL) & - & - & - & - & $\mathrm{x}$ & - & - & - & - & $x$ \\
\hline Galactia striata (Jacq.) Urb. (XAL) 16656 & _ & - & - & - & - & - & $\mathrm{x}$ & _ & $\mathrm{x}$ & - \\
\hline Gliricidia sepium (Jacq.) Steud. (XAL) & - & - & - & $\mathrm{x}$ & - & - & - & - & - & $x$ \\
\hline Indigofera miniata Ortega (XAL) & - & - & - & - & - & $\mathrm{x}$ & - & - & - & $x$ \\
\hline Indigofera mucronata Spreng. ex DC. (XAL) 19300 & - & - & - & - & - & $\mathrm{x}$ & - & - & - & $x$ \\
\hline Indigofera suffruticosa Mill. (XAL) & - & - & - & - & $\mathrm{x}$ & - & - & - & - & $\mathrm{x}$ \\
\hline \multicolumn{11}{|l|}{ Leucaena diversifolia (Schltdl.) Benth. subsp. } \\
\hline diversifolia (XAL) & - & - & - & $\mathrm{x}$ & - & - & - & - & - & $\mathrm{x}$ \\
\hline Leucaena lanceolata S.Watson (XAL) 19441 & - & - & - & $\mathrm{x}$ & - & - & - & - & - & $\mathrm{x}$ \\
\hline Lysiloma acapulcense (Kunth) Benth. (XAL) 17195 & - & - & - & $\mathrm{x}$ & - & - & - & - & $\mathrm{x}$ & - \\
\hline \multirow{2}{*}{\multicolumn{11}{|c|}{ Macroptilium atropurpureum (Sessé et Moc. ex DC.) }} \\
\hline & & & & & & & & & & \\
\hline Urb. (XAL) 19356 & _ & - & - & - & _ & - & $\mathrm{x}$ & _ & - & $\mathrm{x}$ \\
\hline \multicolumn{11}{|l|}{ Mimosa albida Humb. et Bonpl. ex Willd. (XAL) } \\
\hline 18203 & _ & - & - & - & $\mathrm{x}$ & - & - & _ & - & $\mathrm{x}$ \\
\hline Mimosa nelsonii B.L.Rob. (XAL) & - & - & - & - & $x$ & - & - & - & - & $x$ \\
\hline Mimosa pigra $\mathrm{L} .(\mathrm{XAL})$ & - & _. & - & - & $\mathrm{x}$ & - & - & _ & - & $\mathrm{x}$ \\
\hline Mimosa pudica L. (XAL) & - & - & - & - & $x$ & - & - & - & - & $x$ \\
\hline Mimosa tricephala Cham. et Schltdl. (XAL) 16300 & - & - & - & - & $\mathrm{x}$ & - & - & - & - & $\mathrm{x}$ \\
\hline Nissolia fruticosa Jacq. (XAL) 18234 & - & - & - & - & - & - & $\mathrm{x}$ & - & $\mathrm{x}$ & - \\
\hline Phaseolus sp. (XAL) & - & - & - & - & - & - & $\mathrm{x}$ & - & - & $\mathrm{x}$ \\
\hline Phaseolus vulgaris $\mathrm{L}$. (XAL) & - & - & - & - & - & - & $\mathrm{x}$ & - & - & $\mathrm{x}$ \\
\hline Piscidia piscipula (L.) Sarg. (XAL) & - & - & - & $\mathrm{x}$ & - & - & - & - & - & $x$ \\
\hline \multicolumn{11}{|l|}{ Rhynchosia longeracemosa M.Martens et Galeotii } \\
\hline (XAL) 17165 & - & - & - & - & - & - & $\mathrm{x}$ & - & $\mathrm{x}$ & - \\
\hline
\end{tabular}




\begin{tabular}{|c|c|c|c|c|c|c|c|c|c|c|}
\hline Familia y especie & $\mathrm{C}$ & EM & EV & A & $\operatorname{Ar}$ & $\mathrm{H}$ & B & $\mathrm{E}$ & $\mathrm{P}$ & $S$ \\
\hline Rhynchosia minima (L.) DC. (XAL) 19306 & - & - & - & - & - & - & $x$ & - & - & $x$ \\
\hline \multicolumn{11}{|l|}{ Senna atomaria (L.) H.S.Irwin et Barneby (XAL) } \\
\hline 16675 & - & - & - & $\mathrm{X}$ & - & - & - & - & $\mathrm{X}$ & - \\
\hline \multicolumn{11}{|l|}{ Senna cobanensis (Britton) H.S.Irwin et } \\
\hline Barneby (XAL) & - & - & - & - & - & $\mathrm{x}$ & - & - & - & $\mathrm{x}$ \\
\hline \multicolumn{11}{|l|}{ Senna hirsuta (L.) H.S.Irwin et Barneby var. hirta } \\
\hline H.S.Irwin et Barneby (XAL) & - & - & - & - & - & $\mathrm{X}$ & - & - & - & $\mathrm{x}$ \\
\hline Senna occidentalis (L.) Link (XAL) 16704 & - & - & - & - & $\mathrm{x}$ & - & - & - & $\mathrm{X}$ & - \\
\hline \multicolumn{11}{|l|}{ Senna pendula (Willd.) H.S. Irwin \& Barneby (XAL) } \\
\hline 17094 & - & - & - & - & $\mathrm{x}$ & - & - & - & - & $\mathrm{x}$ \\
\hline \multicolumn{11}{|l|}{ Senna racemosa (Mill.) H.S.Irwin et Barneby var. } \\
\hline Stizolobium pruriens (L.) Medik. (XAL) 19433 & - & - & - & - & - & - & $x$ & - & $x$ & - \\
\hline Stylosanthes humilis Kunth (XAL) 19311 & - & - & - & - & - & $\mathrm{x}$ & - & - & - & $\mathrm{x}$ \\
\hline Tephrosia sp. (XAL) & - & - & - & - & - & $\mathrm{x}$ & - & - & - & $\mathrm{x}$ \\
\hline Tephrosia vicioides Schltdl. (XAL) 19105 & - & - & - & - & - & $\mathrm{x}$ & - & - & - & $\mathrm{x}$ \\
\hline Teramnus uncinatus (L.) Sw. (XAL) & - & - & - & - & - & - & $\mathrm{x}$ & - & - & $\mathrm{x}$ \\
\hline Zapoteca formosa (Kunth) H.M.Hern. (XAL) 19406 & - & - & - & - & $\mathrm{x}$ & - & - & - & $\mathrm{X}$ & - \\
\hline Zapoteca lambertiana (G.Don) H.M.Hern. (XAL) & - & - & - & - & $\mathrm{x}$ & - & - & - & - & $\mathrm{x}$ \\
\hline Zornia reticulata Sm. (XAL) 17007 & - & - & - & - & - & $x$ & - & - & - & $\mathrm{x}$ \\
\hline \multicolumn{11}{|l|}{ FAGACEAE } \\
\hline Quercus oleoides Schltdl. et Cham. (XAL) 16985 & - & - & - & $\mathrm{X}$ & - & - & - & - & $\mathrm{X}$ & - \\
\hline \multicolumn{11}{|l|}{ FLACOURTIACEAE } \\
\hline Casearia corymbosa Kunth (XAL) 16414 & - & - & - & $\mathrm{x}$ & - & - & - & - & $\mathrm{x}$ & - \\
\hline Xylosma flexuosa (Kunth) Hemsl. (XAL) 18199 & - & - & - & $\mathrm{x}$ & - & - & - & - & $\mathrm{X}$ & - \\
\hline \multicolumn{11}{|l|}{ Xylosma velutina (Tul.) Triana et Planch. (XAL) } \\
\hline 16407 & - & - & - & - & $x$ & - & - & - & - & $x$ \\
\hline \multicolumn{11}{|l|}{ HERNANDIACEAE } \\
\hline Gyrocarpus jatrophifolius Domin (XAL) 16551 & - & - & - & $\mathrm{x}$ & - & - & - & - & $\mathrm{x}$ & - \\
\hline \multicolumn{11}{|l|}{ HIPPOCRATEACEAE } \\
\hline Hippocratea celastroides Kunth (XAL) 16559 & - & - & - & - & $x$ & - & - & - & $x$ & - \\
\hline \multicolumn{11}{|l|}{ LABIATAE } \\
\hline Hyptis albida Kunth (XAL) 19227 & - & - & - & - & $x$ & - & - & - & $\mathrm{X}$ & - \\
\hline Hyptis pectinata (L.) Poit. (XAL) 16633 & - & - & - & - & - & $\mathrm{x}$ & - & - & $\mathrm{x}$ & - \\
\hline Hyptis suaveolens (L.) Poit. (XAL) 19347 & - & - & - & - & - & $\mathrm{x}$ & - & - & - & $\mathrm{x}$ \\
\hline Hyptis tomentosa Poit. (XAL) & - & - & - & - & $\mathrm{x}$ & - & - & - & - & $\mathrm{x}$ \\
\hline Hyptis verticillata Jacq. (XAL) 19254 & - & - & - & - & - & $x$ & - & - & - & $\mathrm{x}$ \\
\hline Ocimum micranthum Willd. (XAL) 17511 & - & - & - & - & - & $\mathrm{x}$ & - & - & - & $\mathrm{x}$ \\
\hline Salvia coccinea L.f. (XAL) 18081 & - & - & - & - & - & $\mathrm{x}$ & - & - & $\mathrm{X}$ & - \\
\hline Salvia misella Kunth (XAL) 18949 & - & - & - & - & - & $x$ & - & - & - & $\mathrm{x}$ \\
\hline Salvia sp. (XAL) 16493 & - & - & - & - & - & $\mathrm{x}$ & - & - & - & $\mathrm{x}$ \\
\hline Salvia sp. (XAL) 17066 & - & - & - & - & - & $x$ & - & - & $x$ & - \\
\hline Stachys boraginoides Schltdl. et Cham. (XAL) & - & - & - & - & $x$ & - & - & - & - & $x$ \\
\hline \multicolumn{11}{|l|}{ LAURACEAE } \\
\hline Licaria misantlae (Brandegee) Kosterm. (XAL) & - & - & - & $x$ & - & - & - & - & - & $x$ \\
\hline \multicolumn{11}{|l|}{ LOASACEAE } \\
\hline Gronovia scandens L. (XAL) 18003 & - & - & - & - & - & - & $\mathrm{x}$ & - & - & $\mathrm{x}$ \\
\hline Mentzelia aspera L. (XAL) 18373 & - & - & - & - & - & $\mathrm{x}$ & - & - & - & $\mathrm{x}$ \\
\hline \multicolumn{11}{|l|}{ LOGANIACEAE } \\
\hline Buddleia americana L. (XAL) 17151 & - & - & - & - & $\mathrm{x}$ & - & - & - & $\mathrm{x}$ & - \\
\hline \multicolumn{11}{|l|}{ LORANTHACEAE } \\
\hline Struthanthus crassipes (Oliv.) Eichler (XAL) 17148 & - & - & - & - & - & $\mathrm{x}$ & - & - & $\mathrm{X}$ & - \\
\hline \multicolumn{11}{|l|}{ LYTHRACEAE } \\
\hline Cuphea carthagenensis (Jacq.) J.F.Macbr. (XAL) & & & & & & & & & & \\
\hline 18358 & - & - & - & - & - & $\mathrm{x}$ & - & - & - & $\mathrm{x}$ \\
\hline
\end{tabular}




\section{MALPIGHIACEAE}

Gaudichaudia albida Schltdl. et Cham. (XAL) 17130

Gaudichaudia mcvaughii W.R.Anderson (XAL) 16692

Heteropterys brachiata (L.) DC. (XAL) 16711

Malpighia glabra L. (XAL) 16567

Tetrapterys discolor (G.Mey.) DC. (XAL)

Tetrapterys schiedeana Schltdl. et Cham. (XAL) 18300

\section{MALVACEAE}

Abutilon umbellatum (L.) Sweet (XAL)

Anoda cristata (L) Schltdl. (XAL) 17512

Anoda pedunculosa Hochr. (XAL) 19427

Gaya minutiflora Rose (XAL)

Malvastrum americanum (L.) Torr. (XAL)

Malvastrum coromandelianum (L.) Garcke (XAL) 19320

Malvaviscus arboreus Cav. (XAL) 18169

Malvaviscus sp. (XAL) 17149

Pavonia uniflora (Sessé et Moc.) Fryxell (XAL)

Sida acuta Burm.f. (XAL) 17595

Sida ciliaris $\mathrm{L}$. (XAL)

Sida cordifolia L. (XAL) 19478

Sida glabra Mill. (XAL) 17204

Sida rhombifolia L. (XAL) 19308

Sida sp. (XAL)

Sida ulmifolia Mill. (XAL) 19248

Sida urens $\mathrm{L}$. (XAL)

Wissadula amplissima (L) R.E.Fr. (XAL) 19289

\section{MARTYNIACEAE}

Martynia annua L. (XAL) 18231

\section{MELIACEAE}

Cedrela odorata L. (XAL) 16547

Trichilia havanensis Jacq. (XAL) 16305

Trichilia hirta L. (XAL) 19025

\section{MENISPERMACEAE}

Cissampelos pareira L. (XAL)

\section{MOLLUGINACEAE}

Mollugo verticillata L. (XAL) 19262

MORACEAE

Cecropia obtusifolia Bertol. (XAL) 17107

Ficus aurea Nutt. (XAL)

Ficus cotinifolia Kunth (XAL) 18190

Ficus glycicarpa (Miq.) Miq. (XAL) 17105

Ficus goldmanii Standl. (XAL)

Ficus obtusifolia Kunth (XAL)

Ficus pertusa L.f. (XAL) 16973

Ficus rzedowskii Carvajal et Cuevas-Figueroa (XAL) 18136

\section{MYRSINACEAE}

Ardisia escallonioides Schltdl. et Cham. (XAL) 16963 MYRTACEAE

Calyptranthes schiediana O.Berg (XAL) 17104

Eugenia acapulcensis Steud. (XAL) 16539

Eugenia mozomboensis P.E.Sánchez (XAL) 19505

Psidium guajava L. (XAL) 18486 


\begin{tabular}{|c|c|c|c|c|c|c|c|c|c|c|}
\hline Familia y especie & $\mathrm{C}$ & EM & EV & A & $\operatorname{Ar}$ & $\mathrm{H}$ & B & $\mathrm{E}$ & $\mathrm{P}$ & $\mathrm{s}$ \\
\hline \multicolumn{11}{|l|}{ NYCTAGINACEAE } \\
\hline Boerhavia coccinea Mill. (XAL) & - & - & - & - & - & $\mathrm{x}$ & - & - & $\mathrm{x}$ & - \\
\hline Boerhavia erecta L. (XAL) 19290 & - & - & - & - & - & $\mathrm{x}$ & - & - & - & $\mathrm{x}$ \\
\hline Pisonia aculeata L. (XAL) 17632 & - & - & - & - & $x$ & - & - & - & $x$ & - \\
\hline \multicolumn{11}{|l|}{ OLACACEAE } \\
\hline Schoepfia schreberi J.F.Gmel. (XAL) 17002 & - & $\mathrm{x}$ & - & - & $\mathrm{x}$ & - & - & - & $\mathrm{x}$ & - \\
\hline Ximenia americana L. (XAL) 16993 & - & $\mathrm{x}$ & - & - & $\mathrm{x}$ & - & - & - & $x$ & - \\
\hline \multicolumn{11}{|l|}{ OLEACEAE } \\
\hline Fraxinus schiedeana Schltdl. et Cham. (XAL) 16412 & - & - & - & $x$ & - & - & - & - & $x$ & - \\
\hline \multicolumn{11}{|l|}{ ONAGRACEAE } \\
\hline Ludwigia octovalvis (Jacq.) P.H.Raven (XAL) & - & - & - & - & - & $\mathrm{x}$ & - & - & - & $\mathrm{x}$ \\
\hline \multicolumn{11}{|l|}{ OXALIDACEAE } \\
\hline Oxalis corniculata L. (XAL) 16440 & - & - & - & - & - & $\mathrm{x}$ & - & - & - & $\mathrm{x}$ \\
\hline Oxalis latifolia Kunth (XAL) & - & - & - & - & - & $\mathrm{x}$ & - & - & - & $x$ \\
\hline Oxalis neaei DC. (XAL) 19049 & - & - & - & - & - & $\mathrm{x}$ & - & - & - & $x$ \\
\hline \multicolumn{11}{|l|}{ PAPAVERACEAE } \\
\hline Argemone mexicana L. (XAL) 16464 & - & - & - & - & - & $\mathrm{x}$ & - & - & - & $\mathrm{x}$ \\
\hline Bocconia frutescens L. (XAL) 17124 & - & - & - & - & $x$ & - & - & - & $x$ & - \\
\hline \multicolumn{11}{|l|}{ PASSIFLORACEAE } \\
\hline Passiflora biflora Lam. (XAL) 16712 & - & - & - & - & - & - & $\mathrm{x}$ & - & $\mathrm{x}$ & - \\
\hline Passiflora filipes Benth. (XAL) 18435 & - & - & - & - & - & - & $x$ & - & - & $x$ \\
\hline Passiflora holosericea L. (XAL) 17922 & - & - & - & - & - & - & $\mathrm{x}$ & - & - & $\mathrm{x}$ \\
\hline Passiflora oerstedii Mast. (XAL) 18005 & - & - & - & - & - & - & $x$ & - & - & $x$ \\
\hline Passiflora suberosa Link et Otto (XAL) 18099 & - & - & - & - & - & - & $\mathrm{x}$ & - & - & $\mathrm{x}$ \\
\hline \multicolumn{11}{|l|}{ PHYTOLACCACEAE } \\
\hline Petiveria alliacea L. (XAL) & - & - & - & - & - & $\mathrm{x}$ & - & - & - & $x$ \\
\hline Rivina humilis L. (XAL) 18160 & - & - & - & - & - & $\mathrm{x}$ & - & - & $\mathrm{x}$ & - \\
\hline \multicolumn{11}{|l|}{ PIPERACEAE } \\
\hline Peperomia blanda (Jacq.) Kunth (XAL) 18517 & - & - & - & - & - & $\mathrm{X}$ & - & - & $\mathrm{x}$ & - \\
\hline Peperomia glabella (Sw.) A.Dietr. (XAL) 16616 & - & - & - & - & - & $\mathrm{x}$ & - & - & $\mathrm{x}$ & - \\
\hline Peperomia granulosa Trel. (XAL) 19152 & - & - & - & - & - & $\mathrm{x}$ & - & - & $\mathrm{x}$ & - \\
\hline Peperomia obtusifolia (L.) A.Dietr. (XAL) 19122 & - & - & - & - & - & $\mathrm{x}$ & - & - & $x$ & - \\
\hline Piper nudum C.DC. (XAL) 19021 & - & - & - & - & $\mathrm{x}$ & - & - & - & - & $\mathrm{x}$ \\
\hline \multicolumn{11}{|l|}{ PLANTAGINACEAE } \\
\hline Plantago scariosa E.Morris (XAL) 16854 & - & - & - & - & - & $\mathrm{X}$ & - & - & $\mathrm{x}$ & - \\
\hline \multicolumn{11}{|l|}{ PLUMBAGINACEAE } \\
\hline Plumbago scandens L. (XAL) & - & - & - & - & - & $\mathrm{X}$ & - & - & $\mathrm{x}$ & - \\
\hline \multicolumn{11}{|l|}{ POLYGALACEAE } \\
\hline Polygala rivinifolia Kunth (XAL) 18507 & - & - & - & - & - & $\mathrm{x}$ & - & - & $\mathrm{x}$ & - \\
\hline \multicolumn{11}{|l|}{ POLYGONACEAE } \\
\hline \multicolumn{11}{|l|}{ Antigonon cinerascens M.Martens et Galeotii (XAL) } \\
\hline 16637 & - & - & - & - & - & - & $\mathrm{x}$ & - & - & $\mathrm{x}$ \\
\hline Coccoloba liebmanni Lindau (XAL) 16545 & - & - & - & $\mathrm{x}$ & - & - & - & - & - & $x$ \\
\hline Polygonum hydropiperoides Michx. (XAL) 19263 & - & - & - & - & - & $\mathrm{X}$ & - & - & - & $\mathrm{x}$ \\
\hline Ruprechtia fusca Fernald (XAL) 18378 & - & - & - & $\mathrm{x}$ & - & - & - & - & - & $x$ \\
\hline \multicolumn{11}{|l|}{ PORTULACACEAE } \\
\hline Portulaca oleracea L. (XAL) 17047 & - & - & - & - & - & $\mathrm{x}$ & - & - & - & $\mathrm{x}$ \\
\hline Portulaca pilosa L. (XAL) 17628 & - & - & - & - & - & $\mathrm{x}$ & - & - & - & $\mathrm{x}$ \\
\hline Talinum paniculatum (Jacq.) Gaertn. (XAL) 18264 & - & - & - & - & - & $\mathrm{x}$ & - & - & $\mathrm{x}$ & - \\
\hline Talinum triangulare (Jacq.) Willd. (XAL) 19200 & - & - & - & - & - & $\mathrm{x}$ & - & - & - & $x$ \\
\hline \multicolumn{11}{|l|}{ RANUNCULACEAE } \\
\hline Clematis dioica L. (XAL) & - & - & - & - & - & - & $\mathrm{x}$ & - & - & $\mathrm{x}$ \\
\hline Clematis grossa Benth. (XAL) & - & - & - & - & - & - & $\mathrm{x}$ & - & - & $\mathrm{x}$ \\
\hline RHAMNACEAE & & & & & & & & & & \\
\hline Colubrina triflora Brongn. ex. Sweet (XAL) 19438 & - & - & - & $\mathrm{x}$ & - & - & - & - & $\mathrm{x}$ & - \\
\hline
\end{tabular}




\begin{tabular}{|c|c|c|c|c|c|c|c|c|c|c|}
\hline Familia y especie & $\mathrm{C}$ & EM & EV & A & $\mathrm{Ar}$ & $\mathrm{H}$ & B & $\mathrm{E}$ & $\mathrm{P}$ & $\mathrm{S}$ \\
\hline Gouania lupuloides (L.) Urb. (XAL) & - & - & - & - & - & - & $\mathrm{x}$ & - & $\mathrm{x}$ & - \\
\hline \multicolumn{11}{|l|}{ Karwinskia humboldtiana (Willd. ex Roem. et Schult.) } \\
\hline Zucc. (XAL) 16306 & - & - & - & - & $\mathrm{x}$ & - & - & - & - & $\mathrm{x}$ \\
\hline Sageretia elegans (Kunth) Brongn. (XAL) 17157 & - & - & - & - & $x$ & - & - & - & $\mathrm{x}$ & - \\
\hline \multicolumn{11}{|l|}{ RUBIACEAE } \\
\hline Borreria laevis (Lam.) Griseb. (XAL) 19495 & - & - & - & - & - & $\mathrm{x}$ & - & - & - & $\mathrm{x}$ \\
\hline Bouvardia ternifolia (Cav.) Schltdl. (XAL) 16803 & - & - & - & - & $\mathrm{x}$ & - & - & - & $\mathrm{x}$ & - \\
\hline Chiococca alba (L.) Hitchc. (XAL) 18145 & - & - & - & - & $x$ & - & - & - & $\mathrm{x}$ & - \\
\hline Diodia erecta Sessé et Moc. (XAL) & - & - & - & - & - & $\mathrm{x}$ & - & - & - & $\mathrm{x}$ \\
\hline Diodia teres Walter (XAL) 19448 & - & - & - & - & - & $\mathrm{x}$ & - & - & - & $x$ \\
\hline Guettarda elliptica Sw. (XAL) 19271 & - & - & - & - & $x$ & - & - & - & $x$ & - \\
\hline Hamelia patens Jacq. (XAL) 17556 & - & - & - & - & $x$ & - & - & - & $\mathrm{x}$ & - \\
\hline Mitracarpus hirtus (L.) DC. (XAL) & - & - & - & - & - & $\mathrm{x}$ & - & - & - & $x$ \\
\hline Psychotria erythrocarpa Schltdl. (XAL) 16313 & - & - & - & - & $x$ & - & - & - & $\mathrm{x}$ & - \\
\hline Randia aculeata L. (XAL) 16538 & - & - & - & - & $x$ & - & - & - & - & $\mathrm{x}$ \\
\hline Randia monantha Benth. (XAL) 18329 & - & - & - & $\mathrm{x}$ & - & - & - & - & $\mathrm{x}$ & - \\
\hline Randia xalapensis M.Martens et Galeotii (XAL) 19467 & - & - & - & - & $x$ & - & - & - & $\mathrm{x}$ & - \\
\hline Richardia scabra L. (XAL) & - & - & - & - & - & $x$ & - & - & - & $x$ \\
\hline Spermacoce assurgens Ruiz et Pav. (XAL) & - & - & - & - & - & $\mathrm{x}$ & - & - & - & $x$ \\
\hline Spermacoce confusa Rendle (XAL) & - & - & - & - & - & $\mathrm{x}$ & - & - & - & $\mathrm{x}$ \\
\hline Spermacoce sp. (XAL) 16685 & - & - & - & - & - & $\mathrm{x}$ & - & - & $x$ & - \\
\hline Spermacoce tenuior L. (XAL) & - & - & - & - & - & $\mathrm{x}$ & - & - & - & $\mathrm{x}$ \\
\hline Spermacoce tetraquetra A.Rich. (XAL) & - & - & - & - & - & $\mathrm{x}$ & - & - & - & $x$ \\
\hline \multicolumn{11}{|l|}{ RUTACEAE } \\
\hline Amyris purpusii P.Wilson (XAL) 17116 & - & - & - & $\mathrm{x}$ & - & - & - & - & $x$ & - \\
\hline \multirow{2}{*}{\multicolumn{11}{|c|}{ SAPINDACEAE }} \\
\hline & & & & & & & & & & \\
\hline Cardiospermum grandiflorum Sw. (XAL) & - & - & - & - & - & - & $\mathrm{x}$ & - & $\mathrm{x}$ & - \\
\hline Cardiospermum halicacabum L. (XAL) & - & - & - & - & - & - & $\mathrm{x}$ & - & $\mathrm{x}$ & - \\
\hline Dodonaea viscosa Jacq. (XAL) 18100 & - & - & - & - & $x$ & - & - & - & $\mathrm{x}$ & - \\
\hline Exothea paniculata (Juss.) Radlk. (XAL) 17911 & - & - & - & - & $x$ & - & - & - & $x$ & - \\
\hline Paullinia costaricensis Radlk. (XAL) 16694 & - & - & - & - & - & - & $\mathrm{x}$ & - & $\mathrm{x}$ & - \\
\hline Paullinia fuscescens Kunth (XAL) 17155 & - & - & - & - & - & - & $x$ & - & $x$ & - \\
\hline Paullinia sp. (XAL) 17639 & - & - & - & - & - & - & $x$ & - & - & $x$ \\
\hline Paullinia tomentosa Jacq. (XAL) & - & - & - & - & - & - & $\mathrm{x}$ & - & $\mathrm{x}$ & - \\
\hline Serjania cardiospermoides Schltdl. et Cham. & - & - & - & - & - & - & $\mathrm{x}$ & - & - & $x$ \\
\hline Serjania racemosa Schumach. (XAL) 17097 & - & - & - & - & - & - & $x$ & - & - & $x$ \\
\hline Serjania sp. (XAL) 18284 & - & - & - & - & - & $x$ & - & - & - & $x$ \\
\hline Serjania triquetra Radlk. (XAL) 19348 & - & - & - & - & - & - & $\mathrm{x}$ & - & - & $\mathrm{x}$ \\
\hline Urvillea ulmacea Kunth (XAL) & - & - & - & - & - & - & $\mathrm{x}$ & - & - & $\mathrm{x}$ \\
\hline \multicolumn{11}{|l|}{ SAPOTACEAE } \\
\hline Bumelia celastrina Kunth (XAL) 18201 & - & - & - & $\mathrm{x}$ & - & - & - & - & $\mathrm{x}$ & - \\
\hline \multicolumn{11}{|l|}{ SCROPHULARIACEAE } \\
\hline Angelonia sp. (XAL) & - & - & - & - & - & $\mathrm{x}$ & - & & - & $\mathrm{x}$ \\
\hline Bacopa procumbens (Mill.) Greenm. (XAL) & - & - & - & - & - & $\mathrm{x}$ & - & - & - & $x$ \\
\hline Capraria biflora L. (XAL) & - & - & - & - & - & $x$ & - & - & - & $x$ \\
\hline Conobea pusilla Benth. et Hook.f. (XAL) 16682 & - & - & - & - & - & $\mathrm{x}$ & - & - & $\mathrm{x}$ & - \\
\hline Russelia coccinea (L.) Wettst. (XAL) 16631 & - & - & - & - & - & $\mathrm{x}$ & - & - & $\mathrm{x}$ & - \\
\hline Schistophragma pusillum Benth. & - & - & - & - & - & $\mathrm{x}$ & - & - & - & $x$ \\
\hline Stemodia verticillata (Mill.) HassI. (XAL) 19261 & - & - & - & - & - & $\mathrm{x}$ & - & - & - & $x$ \\
\hline \multicolumn{11}{|l|}{ SIMAROUBACEAE } \\
\hline \multirow{2}{*}{\multicolumn{11}{|c|}{$\begin{array}{l}\text { SOLANACEAE } \\
\text { SOLA }\end{array}$}} \\
\hline & & & & & & & & & & \\
\hline Capsicum annuum L. (XAL) 18164 & - & - & - & - & $\mathrm{x}$ & - & - & - & $x$ & - \\
\hline Cestrum dumetorum Schltdl. (XAL) 17096 & - & - & - & - & $\mathrm{x}$ & - & - & - & - & $\mathrm{x}$ \\
\hline
\end{tabular}




\begin{tabular}{|c|c|c|c|c|c|c|c|c|c|c|}
\hline Familia y especie & $\mathrm{C}$ & EM & $\mathrm{EV}$ & A & $\operatorname{Ar}$ & $\mathrm{H}$ & B & $\mathrm{E}$ & $\mathrm{P}$ & S \\
\hline Lycianthes lenta (Cav.) Bitter (XAL) 19513 & - & - & - & - & $\mathrm{x}$ & - & - & - & $\mathrm{x}$ & - \\
\hline Nicotiana plumbaginifolia Viv. (XAL) 19264 & - & - & - & - & - & $\mathrm{x}$ & - & - & - & $\mathrm{x}$ \\
\hline Physalis cinerascens (Dunal) Hitchc. (XAL) 18925 & - & - & - & - & - & $\mathrm{x}$ & - & - & - & $\mathrm{x}$ \\
\hline Physalis philadelphica Lam. (XAL) 18622 & - & - & - & - & - & $\mathrm{x}$ & - & - & - & $\mathrm{x}$ \\
\hline Solanum adscendens Sendtn. (XAL) 18800 & - & - & - & - & - & $\mathrm{x}$ & - & - & - & $\mathrm{x}$ \\
\hline Solanum americanum Mill. (XAL) 16369 & - & - & - & - & - & $\mathrm{x}$ & - & - & - & $\mathrm{x}$ \\
\hline Solanum lanceolatum Cav. (XAL) 18740 & - & - & - & - & $x$ & - & - & - & - & $\mathrm{x}$ \\
\hline Solanum sp. (XAL) 18612 & - & - & - & - & $\mathrm{x}$ & - & - & - & - & $\mathrm{x}$ \\
\hline Solanum tridynamum Dunal (XAL) 17620 & - & $\mathrm{x}$ & - & - & $\mathrm{x}$ & - & - & - & - & $\mathrm{x}$ \\
\hline Solanum umbellatum Mill. (XAL) 18152 & - & - & - & - & $\mathrm{x}$ & - & - & - & - & $\mathrm{x}$ \\
\hline \multicolumn{11}{|l|}{ STERCULIACEAE } \\
\hline Ayenia standleyi Cristóbal (XAL) 18241 & - & - & - & - & - & $\mathrm{x}$ & - & - & $\mathrm{x}$ & - \\
\hline Guazuma ulmifolia Lam. (XAL) 19013 & - & - & - & $x$ & - & - & - & - & - & $\mathrm{x}$ \\
\hline Melochia nodiflora Sw. (XAL) 18235 & - & - & - & - & - & $\mathrm{X}$ & - & - & - & $\mathrm{x}$ \\
\hline Melochia pyramidata L. (XAL) 18289 & - & - & - & - & - & $\mathrm{x}$ & - & - & - & $\mathrm{x}$ \\
\hline Waltheria americana L. & - & - & - & - & $\mathrm{x}$ & - & - & - & $\mathrm{x}$ & - \\
\hline Waltheria indica L. (XAL) 16627 & - & - & - & - & $\mathrm{x}$ & - & - & - & $\mathrm{x}$ & - \\
\hline \multicolumn{11}{|l|}{ THEOPHRASTACEAE } \\
\hline \multicolumn{11}{|l|}{ Jacquinia macrocarpa Cav. subsp. macrocarpa (XAL) } \\
\hline 14847 & - & - & - & - & $x$ & - & - & - & - & $\mathrm{x}$ \\
\hline \multicolumn{11}{|l|}{ TILIACEAE } \\
\hline Corchorus siliquosus L. (XAL) 19034 & - & - & - & - & - & $\mathrm{x}$ & - & - & - & $\mathrm{x}$ \\
\hline Heliocarpus americanus L. (XAL) 18527 & - & - & - & $\mathrm{x}$ & - & - & - & - & $\mathrm{x}$ & - \\
\hline Heliocarpus donnellsmithii Rose & - & - & - & $\mathrm{x}$ & - & - & - & - & $\mathrm{x}$ & - \\
\hline Heliocarpus mexicanus (Turcz.) Sprague (XAL) 17141 & - & - & - & $x$ & - & - & - & - & $\mathrm{X}$ & - \\
\hline Heliocarpus pallidus Rose (XAL) 16659 & - & - & - & $\mathrm{X}$ & - & - & - & - & $\mathrm{x}$ & - \\
\hline Heliocarpus sp. (XAL) T 17232 & - & - & - & $\mathrm{x}$ & - & - & - & - & $\mathrm{x}$ & - \\
\hline \multicolumn{11}{|l|}{ Luehea candida (Moc. et Sessé ex DC.) Mart. (XAL) } \\
\hline 16321 & - & - & - & $\mathrm{x}$ & - & - & - & - & $\mathrm{x}$ & - \\
\hline Triumfetta sp. (XAL) & - & - & - & $\mathrm{X}$ & - & - & - & - & $\mathrm{x}$ & - \\
\hline Triumfetta semitriloba Jacq. (XAL) 17082 & - & - & - & - & - & $x$ & - & - & - & $\mathrm{x}$ \\
\hline \multicolumn{11}{|l|}{ TURNERACEAE } \\
\hline Turnera sp. (XAL) 16410 & - & - & - & - & - & $x$ & - & - & - & $\mathrm{x}$ \\
\hline \multicolumn{11}{|l|}{ ULMACEAE } \\
\hline Celtis caudata Planch. (XAL) 16574 & - & - & - & $\mathrm{x}$ & - & - & - & - & $\mathrm{x}$ & - \\
\hline Celtis iguanaea (Jacq.) Sarg. (XAL) 16561 & - & - & - & $x$ & - & - & - & - & $\mathrm{x}$ & - \\
\hline Trema micrantha (L.) Blume (XAL) 16647 & - & - & - & $x$ & - & - & - & - & $x$ & - \\
\hline \multicolumn{11}{|l|}{ URTICACEAE } \\
\hline Pilea microphylla (L.) Liebm. (XAL) 18236 & - & - & - & - & - & $\mathrm{x}$ & - & - & $\mathrm{x}$ & - \\
\hline \multicolumn{11}{|l|}{ Urera caracasana (Jacq.) Gaudich. ex Griseb (XAL) } \\
\hline 18321 & - & - & - & $\mathrm{X}$ & - & - & - & - & - & $\mathrm{x}$ \\
\hline \multicolumn{11}{|l|}{ VERBENACEAE } \\
\hline Bouchea prismatica (L.) Kuntze (XAL) & - & - & - & - & - & $\mathrm{x}$ & - & - & - & $\mathrm{x}$ \\
\hline Duranta repens L. (XAL) 18523 & - & - & - & - & $\mathrm{x}$ & - & - & - & - & $\mathrm{x}$ \\
\hline Lantana achyranthifolia Desf. (XAL) 18228 & - & - & - & - & $x$ & - & - & - & - & $\mathrm{x}$ \\
\hline Lantana camara L. (XAL) 19024 & - & - & - & - & $x$ & - & - & - & $\mathrm{X}$ & - \\
\hline Lantana hirta Graham (XAL) 17097 B & - & - & - & - & $x$ & - & - & - & $\mathrm{x}$ & - \\
\hline Lippia myriocephala Schltdl. et Cham. (XAL) 17172 & - & - & - & - & - & $\mathrm{x}$ & - & - & $\mathrm{x}$ & - \\
\hline Lippia nodiflora (L.) Michx. (XAL) 19255 & - & - & - & - & - & $\mathrm{x}$ & - & - & - & $\mathrm{x}$ \\
\hline Petrea volubilis L. (XAL) 19463 & - & - & - & - & - & - & $\mathrm{x}$ & - & - & $\mathrm{x}$ \\
\hline Priva lappulacea (L.) Pers. (XAL) & - & - & - & - & - & $\mathrm{x}$ & - & - & - & $\mathrm{x}$ \\
\hline Tamonea curassavica (L.) Pers. (XAL) 18028 & - & - & - & - & - & $\mathrm{x}$ & - & - & - & $\mathrm{x}$ \\
\hline Verbena litoralis Kunth (XAL) 16903 & - & - & - & - & - & $x$ & - & - & - & $x$ \\
\hline
\end{tabular}


Gonzalo Castillo-Campos, Patricia Dávila-Aranda y José Alejandro Zavala-Hurtado

\begin{tabular}{|c|c|c|c|c|c|c|c|c|c|c|}
\hline Familia y especie & C & EM & EV & A & $\mathrm{Ar}$ & $\mathrm{H}$ & $\mathrm{B}$ & $\mathrm{E}$ & $\mathrm{P}$ & $\mathrm{S}$ \\
\hline \multicolumn{11}{|l|}{ VIOLACEAE } \\
\hline $\begin{array}{l}\text { Hybanthus attenuatus (Humb. et Bonpl. ex Roem. } \\
\text { et Schult.) Schulze-Menz (XAL) }\end{array}$ & - & - & - & - & - & $x$ & - & - & - & $x$ \\
\hline \multicolumn{11}{|l|}{ VISCACEAE } \\
\hline $\begin{array}{l}\text { Phoradendron quadrangulare (Kunth) Krug et Urb. } \\
\text { (XAL) } 19056\end{array}$ & - & - & - & $\mathrm{x}$ & - & - & - & - & $\mathrm{X}$ & - \\
\hline \multicolumn{11}{|l|}{ VITACEAE } \\
\hline Cissus rhombifolia Vahl (XAL) 16642 & - & - & - & - & - & - & $\mathrm{x}$ & - & $\mathrm{x}$ & - \\
\hline Cissus sicyoides L. (XAL) 18334 & - & - & - & - & - & - & $\mathrm{x}$ & - & - & $\mathrm{x}$ \\
\hline \multicolumn{11}{|l|}{ ZYGOPHYLLACEAE } \\
\hline Kallstroemia maxima (L.) Hook. et Arn. (XAL) 18165 & - & - & - & - & - & $\mathrm{x}$ & - & - & - & $\mathrm{X}$ \\
\hline
\end{tabular}

